\title{
Stabilized mixed finite elements for deformable porous media with double porosity
}

\author{
Jinhyun Choo · Ronaldo I. Borja* \\ Department of Civil and Environmental Engineering, Stanford University, \\ Stanford, CA 94305, USA. \\ ${ }^{*}$ Corresponding author, E-mail: borja@stanford.edu
}

Summary. Natural geomaterials such as fissured rocks and aggregated soils often exhibit a pore size distribution with two dominant pore scales, usually termed macropores and micropores. High-fidelity descriptions of these materials require an explicit treatment of the two pore regions as double porosity. In this paper, we present stabilized mixed finite elements for coupled flow and deformation in porous media with double porosity based on a thermodynamically consistent modeling framework. Mixed finite elements that interpolate the solid displacement and pore pressures in the macropores and micropores are used for this purpose. In the limit of undrained deformation, the two pore pressures become incompressibility constraints that cause an inf-sup instability in the form of spurious oscillation at the two pore scales. To circumvent this instability we develop a variant of the polynomial pressure projection technique for a twofold saddle point problem. The proposed stabilization allows the use of equal-order (linear) interpolations of the displacement and two pore pressure variables throughout the entire range of drainage condition.

Keywords: coupled problem - double porosity - effective stress - mixture theory $\cdot$ stabilized finite elements $\cdot$ twofold saddle point problem

\section{Introduction}

Natural geomaterials often exhibit a pore size distribution with two dominant pore scales, usually termed macropores and micropores. Examples include fissured rocks and aggregated soils. In fissured rocks the macropores and micropores are the fissures and matrix pores, respectively, whereas in aggregated soils they are the inter-aggregate and intra-aggregate pores. Due to the significant difference in pore sizes, the two pore regions exhibit highly contrasting hydromechanical responses. For example, fissures in rocks serve as conduits for fluid flow that can significantly influence the preferential flow patterns, whereas the pores in the matrix can also provide substantial space 
for fluid transport and storage $[9,35,48,64,94]$. In aggregated soils, the interaggregate pores form the primary pathways for rapid infiltration, but fluids can also migrate through the contact surfaces between the aggregates, as well as within the aggregates [22, 47, 75]. A number of studies have emphasized that high-fidelity modeling of such materials require an explicit consideration of fluid flow and/or solid deformation occurring at the two scales (see [1, 27$29,36,46,49,54,60,62,68,74,79,85-87,93]$ for some of the most recent work).

"Double porosity" is a conceptual framework for a continuum description of porous materials exhibiting two dominant pore scales. In the double porosity framework, the two pore scales are treated as superimposed and interacting domains with explicit representations of the two regions through their volume fractions. A key advantage of the double porosity concept is its capability to handle extremely localized and concentrated flows with relatively simple and homogenized parameters that could otherwise be very complicated to describe, let alone, quantify. Since first proposed in Barenblatt et al. [9], the concept of double porosity has been used extensively for describing fluid flow in fissured/fractured rocks (e.g., [6, 23, 34, 38, 55, 63, 69, 80]) and in aggregated soils (e.g., [21, 22, 72, 92]). Several studies have validated the double porosity framework through homogenization theory [3], laboratory experiments [57, 58], and flow inversion problems [50, 83]. The present study represents one of the very few attempts to formulate the double porosity concept into a robust finite element framework for coupled poromechanics. The paper also proposes a robust stabilization technique in the undrained limit to circumvent the pore pressure oscillation at the two pore scales.

Mathematical modeling of coupled double porosity flow and deformation poses significant challenge in both the theoretical formulation and numerical implementation. On the theoretical side, imposing individual conservation laws for each pore scale is a straightforward way of extending the governing equations to materials with double porosity, as presented in [15]. However, it is less straightforward to develop hydromechanical constitutive relations for double porosity materials. With respect to fluid flow, while Darcy's law may still be applied for transport at each pore scale, an additional relationship is needed to accommodate fluid mass transfer between the two pore regions. For diffusive mass transfer some semi-empirical equations have been advanced in the literature [32, 39, 89].

With respect to solid deformation, on the other hand, an appropriate form of the so-called effective stress tensor should be defined for constitutive modeling of the mechanical behavior. Using principles of thermodynamics and mixture theory, an effective stress tensor that is energy conjugate to the rate of deformation tensor of the solid matrix has been developed in Borja and Koliji [15] for double porosity media. In Koliji et al. [53], a constitutive model for aggregated soils with double porosity has been developed, even as the authors highlighted challenges associated with the measurement of the mi- 
cropore pressures. However, despite these efforts a finite element framework encapsulating all of the essential elements mentioned above remains scarce.

Finite element formulation for a deformable solid with double porosity requires an explicit treatment of the macropore and micropore pressures. This is a natural consequence of the fact that the weighted sum of the two pore pressures represents the overall pore pressure that determines the effective stress [15]. In this paper we pursue a three-field mixed finite element formulation with three primary independent field variables, namely, the solid displacement field, the macropore pressure, and the micropore pressure. Following the standard Galerkin approximation to develop the finite element matrix equation, we arrive at a global coefficient matrix possessing a $3 \times 3$ block structure, in which the pore pressure contributions from the macropores and micropores occupy $2 \times 2$ separate matrix blocks. While this approach may seem to be a natural extension of the single porosity formulation, it is challenged by the mass transfer terms between the two pore scales, as well as by the high contrast in their permeabilities that could trigger numerical instabilities. This motivates an unconditionally stable implicit method for the numerical integration in time domain.

Another form of instability arises in the spatial interpolations of the field variables during undrained deformation when there is no relative flow between the solid and fluid. In the single porosity formulation, this problem gives rise to a coefficient matrix with a $2 \times 2$ block structure form-identical to that encountered in the solution of Stokes flow and incompressible elasticity problems. Instability manifests itself in the form of spurious pressure oscillation, and is widely regarded as the result of the mixed finite element not satisfying the infsup condition $[5,17,18]$. For a thorough discussion of the inf-sup instability in computational poromechanics, we refer the readers to [67]. Equal-order interpolations of displacement and pressure variables are known to exhibit this type of instability, which historically has been the motivation behind the use of higher-order elements such as the Taylor-Hood elements that employ one order higher interpolation for displacement compared to pressure [81]. However, such higher-order interpolation inevitably leads to dramatic increase in the problem size, inhibiting large-scale computations.

To avoid this computational challenge, various stabilization schemes that allow equal-order interpolations of displacement and pressure fields have been developed, including the Brezzi-Pitkäranta scheme [19], the Galerkin leastsquares approach [44], the variational multiscale method [45], and the polynomial pressure projection (PPP) technique [11, 31]. Successful application of these stabilization schemes has been reported for poromechanics of single porosity media $[78,84,88,90]$. However, whereas a few studies have advanced the finite element formulation for double porosity poromechanics (e.g., $[25,33,51,59,73,96])$, none of them has addressed the issue of stabilization.

Due to the presence of two distinct pore scales, stabilization of mixed finite elements for double porosity media is a challenging endeavor. The micropores 
typically exhibit a very low permeability, so they generally deform in nearly undrained fashion; however, the macropores can deform in either undrained or drained mode. A prototype example is fissured rock: the low-permeability rock matrix typically inhibits drainage and only recharges fluids from the fissures, where most of the flow takes place. However, when the rate of loading is fast, both the macropores and micropores can deform in an undrained fashion. With the passage of time, drainage can occur much faster in the macropores than in the micropores, and as a consequence, pressure jumps, or very steep gradients between the macro- and micro-pore pressures, could develop. These pressure jumps enhance the rate of mass transfer between the two pore regions, thereby also affecting their mechanical behavior. Because the two pore pressure fields are treated separately in the proposed double porosity framework, higher-order elements, such as the Taylor-Hood elements, are now even less desirable to use. Low-order mixed finite elements are almost a necessity, thus further motivating the development of robust stabilization techniques for such elements.

In this paper, we present stabilized mixed finite elements for deformable porous media with double porosity. At the core of our stabilization is a variant of the PPP technique, which was initially developed for Stokes flow [11, 31] and later successfully applied to other single-constraint problems including poromechanics $[10,61,78,90]$. We begin with a mixed finite element formulation of coupled flow and deformation in double porosity media based on a thermodynamically consistent modeling framework [25]. Then, we describe a variant of the PPP technique developed for dual treatment of the two pressure constraints in the twofold saddle point problem arising in the undrained limit. The performance and efficacy of the proposed stabilization scheme are demonstrated through several numerical examples involving various combinations of drainage regimes in the two scales. To focus on the topic at hand, namely, stabilization, we shall limit the solid behavior to linear elasticity in the infinitesimal setting. A modeling framework for elastoplastic double porosity materials in the finite deformation range, such as those developed for single porosity problems in $[2,14,16,76,77]$, will be presented in future publications.

\section{Coupled formulation}

This section specializes the double-porosty formulation of [25] for unsaturated porous materials to the fully saturated case, where instability can arise under fully undrained conditions. The presence of air in the pore space induces some compressibility in the solid matrix, so the unsaturated case is not so critical with respect to this type of instability. We use a three-field mixed finite element formulation and highlight the role of the two pore pressure variables in accommodating the volume constraint under undrained condition. 


\subsection{Theory}

Consider a mixture of solid and fluid, in which the fluid fully saturates the solid's macropores and micropores. We use the concept of volume fractions to overlap the solid, fluid in the macropores, and fluid in the micropores:

$$
\phi^{s}=d V_{s} / V, \quad \phi^{i}=d V_{i} / d V, \quad \phi^{s}+\sum_{i=M, m} \phi^{i}=1,
$$

where index $s$ refers to the solid, and index $i$ to the pore scale pertaining to either the macropores $M$ or the micropores $m$. Throughout this paper, we use superscripts to denote partial properties and subscripts to denote intrinsic properties of a constituent.

The partial mass densities can be defined using the volume fractions and intrinsic mass densities as

$$
\rho^{s}=\phi^{s} \rho_{s}, \quad \rho^{i}=\phi^{i} \rho_{f}, \quad \rho=\rho^{s}+\sum_{i=M, m} \rho^{i},
$$

where $\rho$ is the total mass density of the mixture, index $f$ denotes the pore fluid itself, and $\rho$ with upper and lower indices denote partial and intrinsic densities of each constituent, respectively. By definition, the pore fraction for a pore scale is the ratio between the pore volume occupied by this scale to the total volume of the pores in the mixture, i.e.,

$$
\psi^{i}=\frac{\phi^{i}}{1-\phi^{s}}, \quad \sum_{i=M, m} \psi^{i}=1 .
$$

The mechanical behavior of the solid matrix in porous media is governed by the so-called effective stress. In this study we employ a thermodynamically consistent effective stress tensor derived in [15], which is energy-conjugate to the rate of deformation tensor of the solid matrix. Assuming the fluid saturates both the macropores and micropores, the effective Cauchy stress tensor takes the form

$$
\boldsymbol{\sigma}^{\prime}=\boldsymbol{\sigma}+B \bar{p} \mathbf{1}, \quad \bar{p}=\sum_{i=M, m} \psi^{i} p_{i},
$$

where $\boldsymbol{\sigma}$ and $\boldsymbol{\sigma}^{\prime}$ are the total and effective Cauchy stress tensor, $\mathbf{1}$ is the identity tensor, $B$ is the Biot coefficient, $\bar{p}$ is the mean pore pressure for the entire mixture defined as the average of the pore fluid pressures $p_{M}$ and $p_{m}$ weighted according to their pore fractions. The essence of the effective stress concept is to exclude $\bar{p}$ from the total stress, and specific expressions for $\bar{p}$ may depend on the phases and scales of pore fluids. We note that for single porosity continua (i.e., either $\psi^{M}=1$ or $\psi^{m}=1$ ) the effective stress given in (4) reduces to the thermodynamically consistent effective stress derived by Borja [12], which in turn coincides with the Nur and Byerlee [70] effective 
stress in the fully saturated range, and to the Terzaghi effective stress [82] when $B=1$.

For the governing equations we use the balance equations for double porosity continua derived in $[13,15]$. The balance of linear momentum for the entire mixture under quasi-static condition takes the form

$$
\nabla \cdot\left(\boldsymbol{\sigma}^{\prime}-\bar{p} \mathbf{1}\right)+\rho \boldsymbol{g}=\overline{\boldsymbol{c}},
$$

where $\boldsymbol{g}$ is the gravity acceleration vector, and $\overline{\boldsymbol{c}}$ is the momentum exerted by the mass transfer between the macropores and micropores. The mass transfer term is expressed as

$$
\overline{\boldsymbol{c}}=\sum_{i=M, m} c^{i} \tilde{\boldsymbol{v}}_{i}
$$

where $\tilde{\boldsymbol{v}}_{i}$ is the relative velocity of the pore fluid at pore scale $i$ with respect to the solid motion, and $c^{i}$ are mass transfer terms between the two pore scales subject to a closure condition $\sum_{i=M, m} c^{i}=0$. This closure condition is implicitly used to eliminate the solid velocity in (6) from its original expression in $[13,15]$.

The balance of mass for the pore fluids following the solid motion is given by

$$
\frac{\phi^{i}}{K_{f}}\left(\dot{p}_{i}+\tilde{\boldsymbol{v}}_{i} \cdot \nabla p_{i}\right)+\psi^{i} B \nabla \cdot \boldsymbol{v}+\nabla \cdot \boldsymbol{q}_{i}=c^{i}, \quad i=M, m,
$$

where the superimposed dot denotes the material time derivative following the solid motion, $K_{f}$ is the bulk modulus of the pore fluid, $\boldsymbol{v}$ is the solid velocity, $\boldsymbol{q}_{i}=\phi^{i} \tilde{\boldsymbol{v}}_{i}$ is the relative discharge (Darcy) velocity of the pore fluid at pore scale $i$. Without loss of generality, hereafter we assume that the fluids are incompressible and neglect the first term in (7).

Constitutive models for the solid and pore fluids are needed to close the formulation. For the constitutive relation between the effective stress and the deformation of the solid matrix, we use an elastic constitutive model. Defining the infinitesimal strain tensor for the solid matrix as

$$
\boldsymbol{\epsilon}=\nabla^{s} \boldsymbol{u}=\frac{1}{2}(\nabla \boldsymbol{u}+\boldsymbol{u} \nabla),
$$

where $\boldsymbol{u}$ is the displacement of the solid matrix, we write the constitutive stress-strain relationship for the solid matrix in incremental form as

$$
\Delta \sigma^{\prime}=\mathbb{C}: \Delta \epsilon,
$$

where $\mathbb{C}$ is a fourth-order elastic tangent tensor. To accommodate fluid diffusion through the solid matrix, we employ the generalized Darcy's law for isotropic media that takes the form 


$$
\boldsymbol{q}_{i}=-\frac{k_{i}}{\mu_{f}} \cdot\left(\nabla p_{i}-\rho_{f} \boldsymbol{g}\right), \quad i=M, m,
$$

where $k_{i}$ is the intrinsic permeability at pore scale $i$ and $\mu_{f}$ is the dynamic viscosity of the pore fluid.

Assuming the pore fluids in the macropores and micropores are of the same type, we next consider the mass transfer between the two scales by diffusion. In this case, we can introduce a constitutive law that relates the mass transfer term $c^{f}$ with the pressure difference between the two pore regions. A number of expressions have been proposed in the literature accounting for such diffusive mass transfer (e.g., [32, 39, 89]). Here we adopt a semi-empirical equation proposed in [39], given by

$$
c^{M}=\bar{\alpha} \frac{\bar{k}}{\mu_{f}}\left(p_{m}-p_{M}\right), \quad c^{m}=\bar{\alpha} \frac{\bar{k}}{\mu_{f}}\left(p_{M}-p_{m}\right), \quad \bar{\alpha}=\frac{\beta}{a^{2}} \gamma,
$$

where $\bar{k}$ is the interface permeability, $a$ is the characteristic length of the macropores spacing, $\beta$ is a dimensionless coefficient that accounts for the solid matrix geometry, and $\gamma$ is a dimensionless scaling coefficient suggested to be 0.4 to fit experimental results. We note that most other equations suggested in the literature take essentially the same form except for the coefficient $\bar{\alpha}$, and this value also has been estimated from measurements along with inverse analysis [83]. Following [56], we assume that $\bar{k}$ is equal to the permeability of the micropores. Note that the foregoing constitutive expressions automatically satisfy the closure condition $c^{M}+c^{m}=0$.

Remarks on the effective stress. Several expressions for the effective stress $\boldsymbol{\sigma}^{\prime}$ have been proposed in the literature. Many of them have brought substantial progress in micromechanical modeling beyond mixture theories [24, 29, 30, 40, $41,65,95]$. Each expression has its own origin and merits, and, accordingly, some differences in the form of the effective stress exist. Ultimately, the main considerations for choosing a specific form of the effective stress are the scale of the problem and the purpose for which it is intended. The expression for $\boldsymbol{\sigma}^{\prime}$ given in (4) may not allow for detailed micromechanics, but it is good enough for computationally intensive continuum simulations of multiscale and multiphysical problems, where simplicity in form offers a welcome relief.

\subsection{Strong form}

We consider a closed domain denoted by $\bar{\Omega}=\Omega \cup \Gamma$, where $\Omega$ is an open domain and $\Gamma$ is the boundary of $\Omega$. The boundary $\Gamma$ is assumed to be suitably decomposed as: displacement and traction boundaries for solid, $\Gamma_{u}$ and $\Gamma_{t}$, respectively; pressure and flux boundaries for fluid in the macropores, $\Gamma_{p_{M}}$ and $\Gamma_{q_{M}}$, respectively; pressure and flux boundaries for fluid in the micropores, $\Gamma_{p_{m}}$ and $\Gamma_{q_{m}}$, respectively. The boundary relations are 


$$
\begin{aligned}
\Gamma=\overline{\Gamma_{u} \cup \Gamma_{t}} & \text { and } \quad \varnothing=\Gamma_{u} \cap \Gamma_{t} \\
\Gamma=\overline{\Gamma_{p_{M}} \cup \Gamma_{q_{M}}} & \text { and } \quad \varnothing=\Gamma_{p_{M}} \cap \Gamma_{q_{M}} \\
\Gamma=\overline{\Gamma_{p_{m}} \cup \Gamma_{q_{m}}} & \text { and } \quad \varnothing=\Gamma_{p_{m}} \cap \Gamma_{q_{m}}
\end{aligned}
$$

where $\varnothing$ is the null set and the overline denotes a closure.

The strong form of the initial boundary-value problem is as follows. Given $\hat{\boldsymbol{u}}, \hat{\boldsymbol{t}}, \hat{p}_{M}, \hat{q}_{M}, \hat{p}_{m}$, and $\hat{q}_{m}$, find $\boldsymbol{u}, p_{M}$, and $p_{m}$ such that

$$
\nabla \cdot\left(\boldsymbol{\sigma}^{\prime}-\bar{p} \mathbf{1}\right)+\rho \boldsymbol{g}=\overline{\boldsymbol{c}},
$$

and

$$
\psi^{i} B \nabla \cdot \boldsymbol{v}+\nabla \cdot \boldsymbol{q}_{i}=c^{i}, \quad i=M, m,
$$

subject to boundary conditions

$$
\begin{array}{rll}
\boldsymbol{u}=\hat{\boldsymbol{u}} & \text { on } & \Gamma_{u} \\
\boldsymbol{n} \cdot \boldsymbol{\sigma}=\hat{\boldsymbol{t}} & \text { on } & \Gamma_{t} \\
p_{M}=\hat{p}_{M} & \text { on } & \Gamma_{p_{M}} \\
-\boldsymbol{n} \cdot \boldsymbol{q}_{M}=\hat{q}_{M} & \text { on } & \Gamma_{q_{M}} \\
p_{m}=\hat{p}_{m} & \text { on } & \Gamma_{p_{m}} \\
-\boldsymbol{n} \cdot \boldsymbol{q}_{m}=\hat{q}_{m} & \text { on } & \Gamma_{q_{m}},
\end{array}
$$

and initial conditions

$$
\boldsymbol{u}=\boldsymbol{u}_{0}, \quad p_{M}=p_{M 0}, \quad p_{m}=p_{m 0}
$$

for all $(\boldsymbol{x}, t) \in(\bar{\Omega} \times t=0)$. Here, $\boldsymbol{t}$ is the traction vector and the hats denote the prescribed boundary conditions.

\subsection{Weak form}

To develop the variational form of the problem, we first define the spaces of trial functions

$$
\begin{aligned}
\mathcal{S}_{u} & =\left\{\boldsymbol{u} \mid \boldsymbol{u} \in H^{1}, \boldsymbol{u}=\hat{\boldsymbol{u}} \text { on } \Gamma_{u}\right\}, \\
\mathcal{S}_{p_{M}} & =\left\{p_{M} \mid p_{M} \in H^{1}, p_{M}=\hat{p}_{M} \text { on } \Gamma_{p_{M}}\right\}, \\
\mathcal{S}_{p_{m}} & =\left\{p_{m} \mid p_{m} \in H^{1}, p_{m}=\hat{p}_{m} \text { on } \Gamma_{p_{m}}\right\},
\end{aligned}
$$

where $H^{1}$ denotes a Sobolev space of order one. We then introduce the spaces of weighting functions by imposing homogeneous Dirichlet boundary conditions to the corresponding trial function spaces,

$$
\begin{aligned}
\mathcal{V}_{u} & =\left\{\boldsymbol{\eta} \mid \boldsymbol{\eta} \in H^{1}, \boldsymbol{\eta}=\mathbf{0} \text { on } \Gamma_{u}\right\}, \\
\mathcal{V}_{p_{M}} & =\left\{\omega_{M} \mid \omega_{M} \in H^{1}, \omega_{M}=0 \text { on } \Gamma_{p_{M}}\right\}, \\
\mathcal{V}_{p_{m}} & =\left\{\omega_{m} \mid \omega_{m} \in H^{1}, \omega_{m}=0 \text { on } \Gamma_{p_{m}}\right\} .
\end{aligned}
$$


The weak form of the problem is then stated as: Find $\left\{\boldsymbol{u}, p_{M}, p_{m}\right\} \in \mathcal{S}_{u} \times$ $\mathcal{S}_{p_{M}} \times \mathcal{S}_{p_{m}}$ such that for all $\left\{\boldsymbol{\eta}, \omega_{M}, \omega_{m}\right\} \in \mathcal{V}_{u} \times \mathcal{V}_{p_{M}} \times \mathcal{V}_{p_{m}}$ the following equations are satisfied:

(a) Balance of linear momentum

$$
\int_{\Omega} \nabla^{s} \boldsymbol{\eta}:\left(\boldsymbol{\sigma}^{\prime}-B \bar{p} \mathbf{1}\right) \mathrm{d} \Omega=\int_{\Omega} \boldsymbol{\eta} \cdot(\rho \boldsymbol{g}+\overline{\boldsymbol{c}}) \mathrm{d} \Omega+\int_{\Gamma_{t}} \boldsymbol{\eta} \cdot \hat{\boldsymbol{t}} \mathrm{d} \Gamma,
$$

(b) Balance of mass in the macropores

$$
\begin{aligned}
& \int_{\Omega} \omega_{M} \psi^{M} B \nabla \cdot \boldsymbol{v} \mathrm{d} \Omega-\int_{\Omega} \nabla \omega_{M} \cdot \boldsymbol{q}_{M} \mathrm{~d} \Omega \\
& =\int_{\Omega} \omega_{M} c^{M} \mathrm{~d} \Omega+\int_{\Gamma_{q_{M}}} \omega_{M} \hat{q}_{M} \mathrm{~d} \Gamma,
\end{aligned}
$$

(c) Balance of mass in the micropores

$$
\begin{aligned}
& \int_{\Omega} \omega_{m} \psi^{m} B \nabla \cdot \boldsymbol{v} \mathrm{d} \Omega-\int_{\Omega} \nabla \omega_{m} \cdot \boldsymbol{q}_{m} \mathrm{~d} \Omega \\
& =\int_{\Omega} \omega_{m} c^{m} \mathrm{~d} \Omega+\int_{\Gamma_{q_{m}}} \omega_{m} \hat{q}_{m} \mathrm{~d} \Gamma .
\end{aligned}
$$

It is usually more convenient to express the variational equations in timeintegrated form. This applies to the two mass balance equations. Employing the first-order accurate, unconditionally stable backward Euler method, we get the following time-integrated variational equation for the macropores (after multiplying $\Delta t$ ),

$$
\begin{aligned}
& \int_{\Omega} \omega_{M} \psi^{M} B \nabla \cdot\left(\boldsymbol{u}-\boldsymbol{u}_{n}\right) \mathrm{d} \Omega-\Delta t \int_{\Omega} \nabla \omega_{M} \cdot \boldsymbol{q}_{M} \mathrm{~d} \Omega \\
& =\Delta t \int_{\Omega} \omega_{M} c^{M} \mathrm{~d} \Omega+\Delta t \int_{\Gamma_{q_{M}}} \omega_{M} \hat{q}_{M} \mathrm{~d} \Gamma,
\end{aligned}
$$

and the time-integrated variational equation for the micropores,

$$
\begin{aligned}
& \int_{\Omega} \omega_{m} \psi^{m} B \nabla \cdot\left(\boldsymbol{u}-\boldsymbol{u}_{n}\right) \mathrm{d} \Omega-\Delta t \int_{\Omega} \nabla \omega_{m} \cdot \boldsymbol{q}_{m} \mathrm{~d} \Omega \\
& =\Delta t \int_{\Omega} \omega_{m} c^{m} \mathrm{~d} \Omega+\Delta t \int_{\Gamma_{q_{m}}} \omega_{m} \hat{q}_{m} \mathrm{~d} \Gamma,
\end{aligned}
$$

where subscript $n$ denote quantities at time $t_{n}$, and $\Delta t$ denotes a time increment. For brevity in the preceding two equations, we have dropped the subscript " $n+1$ " for quantities pertaining to time $t_{n+1}$. We note that the backward Euler scheme possesses high-frequency numerical damping, so any spurious oscillation in time is automatically suppressed. This is specially desirable for the problem at hand where the two pore scales have a high permeability contrast. 


\subsection{Matrix form}

For the numerical solution of the mixed variational formulation, we consider three-field mixed finite elements with displacement and two pore pressure degrees of freedom at each node (for Taylor-Hood elements some nodes may contain displacement degrees of freedom only). Following the standard Galerkin approximation for spatial discretization, we introduce standard shape function matrices and write

$$
\left.\begin{array}{rl}
\boldsymbol{u}^{h} & =\boldsymbol{N}^{u} \boldsymbol{d}+\hat{\boldsymbol{N}}^{u} \hat{\boldsymbol{d}} \\
p_{M}^{h} & =\boldsymbol{N}^{p} \boldsymbol{p}_{M}+\hat{\boldsymbol{N}}^{p} \hat{\boldsymbol{p}}_{M} \\
p_{m}^{h} & =\boldsymbol{N}^{p} \boldsymbol{p}_{m}+\hat{\boldsymbol{N}}^{p} \hat{\boldsymbol{p}}_{m}
\end{array}\right\},
$$

where superscript $h$ denotes a spatially discretized function; $\boldsymbol{N}$ is the shape function matrix with superscripts indicating the fields being interpolated; $\boldsymbol{d}$, $\boldsymbol{p}_{M}, \boldsymbol{p}_{m}$ are the nodal displacement vector, nodal macropore pressure vector, and nodal micropore pressure vector, respectively; and the hats pertain to contributions from the Dirichlet boundary conditions. Here, we distinguish between the shape functions used for displacement and pore pressure interpolations, and use the same interpolation function for the pressures in the macropores and micropores.

Gradient and divergence of the primary variables can be interpolated accordingly, and here we represent them with the following symbols:

$$
\left.\begin{array}{rl}
\nabla \boldsymbol{u}^{h} & =\boldsymbol{B} \boldsymbol{d}+\hat{\boldsymbol{B}} \hat{\boldsymbol{d}} \\
\nabla \cdot \boldsymbol{u}^{h} & =\boldsymbol{b} \boldsymbol{d}+\hat{\boldsymbol{b}} \hat{\boldsymbol{d}} \\
\nabla p_{M}^{h} & =\boldsymbol{E} \boldsymbol{p}_{M}+\hat{\boldsymbol{E}} \hat{\boldsymbol{p}}_{M} \\
\nabla p_{m}^{h} & =\boldsymbol{E} \boldsymbol{p}_{m}+\hat{\boldsymbol{E}} \hat{\boldsymbol{p}}_{m}
\end{array}\right\} .
$$

The same shape functions are used for the weighting functions.

Substituting the finite elements approximations to the Galerkin form of (30), (33), and (34), we arrive at the following residual equations in vector form

(a) Balance of linear momentum:

$$
\begin{aligned}
\mathcal{R}_{u}^{h}= & -\int_{\Omega}\left(\boldsymbol{B}^{\top} \boldsymbol{\sigma}^{\prime}-\boldsymbol{b}^{\top} B \bar{p}^{h}\right) \mathrm{d} \Omega+\int_{\Omega}\left(\boldsymbol{N}^{u}\right)^{\top}\left(\rho \boldsymbol{g}+\overline{\boldsymbol{c}}^{h}\right) \mathrm{d} \Omega \\
& +\int_{\Gamma_{t}}\left(\boldsymbol{N}^{u}\right)^{\top} \hat{\boldsymbol{t}} \mathrm{d} \Gamma \rightarrow \mathbf{0},
\end{aligned}
$$

(b) Balance of mass in the macropores:

$$
\begin{aligned}
\mathcal{R}_{p_{M}}^{h}= & \int_{\Omega}\left(\boldsymbol{N}^{p}\right)^{\top} \psi^{M} B \nabla \cdot\left(\boldsymbol{u}^{h}-\boldsymbol{u}_{n}^{h}\right) \mathrm{d} \Omega-\Delta t \int_{\Omega} \boldsymbol{E} \cdot \boldsymbol{q}_{M}^{h} \mathrm{~d} \Omega \\
& -\Delta t \int_{\Omega}\left(\boldsymbol{N}^{p}\right)^{\top} c^{M} \mathrm{~d} \Omega-\Delta t \int_{\Gamma_{q_{M}}}\left(\boldsymbol{N}^{p}\right)^{\top} \hat{q}_{M} \mathrm{~d} \Gamma \rightarrow \mathbf{0},
\end{aligned}
$$


(c) Balance of mass in the micropores:

$$
\begin{aligned}
\mathcal{R}_{p_{m}}^{h}= & \int_{\Omega}\left(\boldsymbol{N}^{p}\right)^{\top} \psi^{m} B \nabla \cdot\left(\boldsymbol{u}^{h}-\boldsymbol{u}_{n}^{h}\right) \mathrm{d} \Omega-\Delta t \int_{\Omega} \boldsymbol{E} \cdot \boldsymbol{q}_{m}^{h} \mathrm{~d} \Omega \\
& -\Delta t \int_{\Omega}\left(\boldsymbol{N}^{p}\right)^{\top} c^{m} \mathrm{~d} \Omega-\Delta t \int_{\Gamma_{q_{m}}}\left(\boldsymbol{N}^{p}\right)^{\top} \hat{q}_{m} \mathrm{~d} \Gamma \rightarrow \mathbf{0} .
\end{aligned}
$$

In the foregoing relations, the residual vectors $\mathcal{R}_{(\cdot)}^{h}$ are used for NewtonRaphson iteration until convergence is achieved, as implied by the rightward arrows tending to zero.

In general, the residuals defined above are nonlinear in the primary variables $\boldsymbol{d}, \boldsymbol{p}_{M}$, and $\boldsymbol{p}_{m}$, except when the constitutive laws for the solid deformation and fluid flow are linear. Most porous materials exhibit a nonlinear stress-strain behavior, and even the permeability coefficient may depend on deformation, resulting in a nonlinear flux-gradient relation. To solve for the primary variables in a general nonlinear setting, we make use of a robust Newton-Raphson iteration. To this end, we need to evaluate the consistent tangent operator as follows

$$
\mathcal{R}=\left\{\begin{array}{l}
\mathcal{R}_{u} \\
\mathcal{R}_{p_{M}} \\
\mathcal{R}_{p_{m}}
\end{array}\right\}, \quad \mathcal{R}^{\prime}(X)=\left[\begin{array}{ccc}
A & B_{1} & C_{1} \\
B_{2} & D & E_{1} \\
C_{2} & E_{2} & F
\end{array}\right],
$$

where $X$ is the solution vector with components $\boldsymbol{d}, \boldsymbol{p}_{M}$, and $\boldsymbol{p}_{m}$. Note that the tangent operator has a $3 \times 3$ block structure. The individual matrices comprising the tangent operator are as follows: 


$$
\begin{aligned}
A & =-\int_{\Omega} \boldsymbol{B}^{\top} \boldsymbol{C}_{k} \boldsymbol{B} \mathrm{d} \Omega, \\
B_{1} & =\int_{\Omega} \boldsymbol{b}^{\top} B \psi^{M} \boldsymbol{N}^{p} \mathrm{~d} \Omega+\int_{\Omega}\left(\boldsymbol{N}^{u}\right)^{\top}\left(\frac{\partial \overline{\boldsymbol{c}}^{h}}{\partial p_{M}^{h}}\right) \boldsymbol{N}^{p} \mathrm{~d} \Omega, \\
C_{1} & =\int_{\Omega} \boldsymbol{b}^{\top} B \psi^{m} \boldsymbol{N}^{p} \mathrm{~d} \Omega+\int_{\Omega}\left(\boldsymbol{N}^{u}\right)^{\top}\left(\frac{\partial \overline{\boldsymbol{c}}^{h}}{\partial p_{m}^{h}}\right) \boldsymbol{N}^{p} \mathrm{~d} \Omega, \\
B_{2} & =\int_{\Omega}\left(\boldsymbol{N}^{p}\right)^{\top} B \psi^{M} \boldsymbol{b} \mathrm{d} \Omega, \\
D & =\Delta t \int_{\Omega} \boldsymbol{E}^{\top} \boldsymbol{\kappa}_{M} \boldsymbol{E} \mathrm{d} \Omega-\Delta t \int_{\Omega}\left(\boldsymbol{N}^{p}\right)^{\top}\left(\frac{\partial c^{M}}{\partial p_{M}^{h}}\right) \boldsymbol{N}^{p} \mathrm{~d} \Omega, \\
E_{1} & =-\Delta t \int_{\Omega}\left(\boldsymbol{N}^{p}\right)^{\top}\left(\frac{\partial c^{M}}{\partial p_{m}^{h}}\right) \boldsymbol{N}^{p} \mathrm{~d} \Omega, \\
C_{2} & =\int_{\Omega}\left(\boldsymbol{N}^{p}\right)^{\top} B \psi^{m} \boldsymbol{b} \mathrm{d} \Omega, \\
E_{2} & =-\Delta t \int_{\Omega}\left(\boldsymbol{N}^{p}\right)^{\top}\left(\frac{\partial c^{m}}{\partial p_{M}^{h}}\right) \boldsymbol{N}^{p} \mathrm{~d} \Omega, \\
F & =\Delta t \int_{\Omega} \boldsymbol{E}^{\top} \boldsymbol{\kappa}_{m} \boldsymbol{E} \mathrm{d} \Omega-\Delta t \int_{\Omega}\left(\boldsymbol{N}^{p}\right)^{\top}\left(\frac{\partial c^{m}}{\partial p_{m}^{h}}\right) \boldsymbol{N}^{p} \mathrm{~d} \Omega,
\end{aligned}
$$

where $\boldsymbol{C}_{k}$ is the consistent stress-strain matrix ( $k$ denotes an iteration counter) and $\boldsymbol{\kappa}_{(\cdot)}$ is the matrix of permeability over dynamic viscosity.

We remark that when the diffusive mass transfer is zero, all the derivatives of $c^{M}$ and $c^{m}$ with respect to the two pore pressure variables vanish. In this case, $E_{1}=E_{2}=0, B_{1}=B_{2}^{\top}$, and $C_{1}=C_{2}^{\top}$, leading to a symmetric tangent operator having a form similar to the $2 \times 2$ block matrix arising in single porosity formulation [90]. We also remark that in the limit of undrained deformation, which occurs either when $\Delta t \rightarrow 0$ (fast rate of loading) and/or when $\boldsymbol{\kappa}_{M}, \boldsymbol{\kappa}_{m} \rightarrow \mathbf{0}$ (impermeable solid), combined with the condition of no diffusive mass transfer, the entire $2 \times 2$ lower right-hand side block consisting of submatrices $D, E_{1}, E_{2}$, and $F$ vanishes, resulting in a coefficient matrix that potentially could be either singular (i.e., problem is not solvable) or unstable in the sense of the inf-sup condition for twofold saddle point problems, see [43].

Double porosity formulation could also lead to another interesting scenario, and that is when $\boldsymbol{\kappa}_{m} \rightarrow \mathbf{0}$, which leads to $F \rightarrow 0$. The result is an incompressible micropore but a compressible macropore (if drainage in the macropore is allowed). While this condition may appear to be somewhat similar to that encountered in incompressible elasticity or Stokes flow, in which a diagonal submatrix block also vanishes, the physics of the present problem is not quite the same because of the dual nature of porosity. In any case, whether it is the micropore alone that is incompressible, or it is the entire mixture that cannot change in volume, instabilities in the undrained limit will naturally impact 
the performance of many mixed finite elements. Therefore, this issue must be addressed with a suitable stabilization.

\section{Stabilized mixed finite elements}

The subject of the present stabilization study is the class of low-order mixed finite elements employing equal-order (linear) interpolations of displacement and pressure fields. These elements do not satisfy the inf-sup condition and are known to exhibit pronounced pressure oscillation in the undrained limit. We should note, however, that this class of mixed finite elements generally perform well under drained conditions. Therefore, we seek an effective stabilization that circumvents the pressure oscillation in the undrained limit but preserves the acceptable performance in drained calculations. The added challenge in double porosity stabilization is the high contrast in the permeabilities of the macropores and micropores. The stabilization needs to address potential oscillation in the micropore pressures even if the macropore pressure responses seem stable.

Before considering the double porosity stabilization, we first review the general stability condition for mixed finite elements in single-constraint problems such as Stokes flow, incompressible elasticity, and single porosity poromechanics, for which the stabilization technique extended in this work was originally proposed. The discrete inf-sup condition for velocity and pressure interpolations reads [18]

$$
\sup _{\boldsymbol{v}^{h} \in \mathcal{S}_{u}^{h}} \frac{\int_{\Omega} p^{h} \nabla \cdot \boldsymbol{v}^{h} \mathrm{~d} \Omega}{\left\|\boldsymbol{v}^{h}\right\|_{1}} \geq C\left\|p^{h}\right\|_{0}
$$

for all $p^{h} \in \mathcal{S}_{p}^{h}$, where $\mathcal{S}_{u}^{h}$ and $\mathcal{S}_{p}^{h}$ are the discrete spaces for velocity and pressure interpolations, respectively, $\|\cdot\|_{k}$ is the standard Sobolev norm with order $k$, and $C$ is a positive constant independent of element size $h$. This condition determines the bound for an intrinsically stable pair of finite element spaces $\mathcal{S}_{u}^{h}$ and $\mathcal{S}_{p}^{h}$.

Unfortunately, linear-order pair elements do not satisfy the condition described in the preceding paragraph. However, Bochev et al. [10] showed that they do satisfy a weaker inf-sup condition given by

$$
\sup _{\boldsymbol{v}^{h} \in \mathcal{S}_{u}^{h}} \frac{\int_{\Omega} p^{h} \nabla \cdot \boldsymbol{v}^{h} \mathrm{~d} \Omega}{\left\|\boldsymbol{v}^{h}\right\|_{1}} \geq C_{1}\left\|p^{h}\right\|_{0}-C_{2}\left\|p^{h}-\Pi p^{h}\right\|_{0}
$$

for all $p^{h} \in \mathcal{S}_{p}^{h}$, where $\Pi: L_{2}(\Omega) \rightarrow R_{0}$ is a projection operator from spaces of $L_{2}$ to piecewise constants $R_{0}$, and $C_{1}$ and $C_{2}$ are positive constants independent of $h$. Comparing (50) and (51), we find that the last term, $C_{2}\left\|p^{h}-\Pi p^{h}\right\|_{0}$ in (51), quantifies the deficiency of the equal-order linear pair elements. The idea behind the PPP technique is then to penalize this deficiency by augmenting a stabilization term to the discrete variational equation. 
The projection operator $\Pi$ for the discrete pressure field $p^{h}$ can be defined as

$$
\left.\Pi p^{h}\right|_{\Omega^{e}}=\frac{1}{V^{e}} \int_{\Omega^{e}} p^{h} \mathrm{~d} \Omega,
$$

where $\Omega^{e}$ and $V^{e}$ denote the domain and volume of an element, respectively. In words, the operator $\Pi$ projects a given field to its average value within the element. For single-constraint problems, specific stabilization terms for penalizing the deficiency have essentially taken the same form except for the constants. We emphasize that in all cases the stabilization term should be sufficiently large to penalize the deficiency, but not too large as to be overly diffusive and induce undesirable smoothing.

For single porosity poromechanics, a stabilization term has been proposed by White and Borja [90] for the mass residual term of the form

$$
\mathcal{M}=\int_{\Omega} \frac{1}{2 G}\left(\omega^{h}-\Pi \omega^{h}\right)\left(\overline{p^{h}-\Pi p^{h}}\right) \mathrm{d} \Omega,
$$

where the superimposed dot denotes a time differentiation. After temporal and spatial discretization, the expression becomes

$$
\mathcal{M}^{\prime}=\frac{1}{\Delta t} \int_{\Omega} \frac{1}{2 G}\left[\boldsymbol{N}^{p}-\Pi\left(\boldsymbol{N}^{p}\right)\right]\left[\left(p^{h}-p_{n}^{h}\right)-\Pi\left(p^{h}-p_{n}^{h}\right)\right] \mathrm{d} \Omega,
$$

where $\omega$ and $\boldsymbol{N}^{p}$ are the weighting and shape functions for the pore pressure, respectively, and $G$ is the shear modulus of the solid matrix. We note that the stabilization term originally proposed by Bochev et al. [10] is inherently parameter-free, much like (53) and (54). However, White and Borja [90] introduced a parameter $\tau$ to avoid over-diffusion that may occur when steep pressure gradients are involved, such as in localized zones (e.g., faults) where the permeabilities between adjacent material zones are orders of magnitude different. Recalling that the double porosity framework treats such heterogeneity as different scales, it is very unlikely that similar over-diffusion in the pressure field would be encountered. Therefore, we shall revert back to the original parameter-free PPP technique.

To accommodate two pore pressure constraints, we consider a twofold saddle point problem of the generic form (see [43] for details): Find $\left(u, p_{1}, p_{2}\right) \in$ $\mathcal{S}_{u} \times \mathcal{S}_{p_{1}} \times \mathcal{S}_{p_{2}}$ such that

$$
\begin{aligned}
a(u, v)+b_{1}\left(p_{1}, v\right)+b_{2}\left(p_{2}, v\right) & =f(v) \\
b_{1}\left(q_{1}, u\right) & =g_{1}\left(q_{1}\right) \\
b_{2}\left(q_{2}, u\right) & =g_{2}\left(q_{2}\right),
\end{aligned}
$$

for all $v \in \mathcal{S}_{u}, q_{1} \in \mathcal{S}_{p_{1}}$, and $q_{2} \in \mathcal{S}_{p_{2}}$, where $a, b_{1}$, and $b_{2}$ are bilinear operators. As noted in [43], this can be viewed as a single saddle point problem on $\mathcal{S}_{u} \times\left(\mathcal{S}_{p_{1}} \times \mathcal{S}_{p_{2}}\right)$, with bilinear form 


$$
b\left(\left(p_{1}, p_{2}\right), u\right)=b_{1}\left(p_{1}, u\right)+b_{2}\left(p_{2}, u\right) .
$$

The relevant inf-sup condition is given by (see [43])

$$
\sup _{v \in \mathcal{S}_{u}} \frac{b_{1}\left(v, p_{1}\right)+b_{2}\left(v, p_{2}\right)}{\|v\|_{\mathcal{S}_{u}}} \geq C\left(\left\|p_{1}\right\|_{\mathcal{S}_{p_{1}}}+\left\|p_{2}\right\|_{\mathcal{S}_{p_{2}}}\right)
$$

for all $\left(p_{1}, p_{2}\right) \in \mathcal{S}_{p_{1}} \times \mathcal{S}_{p_{2}}$, with $C>0$.

We are interested in the condition where $b_{1}=b_{2} \equiv b$, and with $\|\cdot\|_{\mathcal{S}_{p_{1}}}=$ $\|\cdot\|_{\mathcal{S}_{p_{2}}} \equiv\|\cdot\|_{\mathcal{S}_{p}}$, which is motivated by the same order of Sobolev spaces for the two pressure fields. In this case, the relevant inf-sup condition, using the triangle inequality, is given by

$$
\begin{aligned}
\sup _{v \in \mathcal{S}_{u}} \frac{b\left(v, p_{1}+p_{2}\right)}{\|v\|_{\mathcal{S}_{u}}} & \geq C\left(\left\|p_{1}\right\|_{\mathcal{S}_{p}}+\left\|p_{2}\right\|_{\mathcal{S}_{p}}\right) \\
& \geq C\left(\left\|p_{1}+p_{2}\right\|_{\mathcal{S}_{p}}\right)
\end{aligned}
$$

for all $\left(p_{1}, p_{2}\right) \in \mathcal{S}_{p} \times \mathcal{S}_{p}$, with $C>0$. This motivates the following stabilization for the problem at hand.

Consider the problem of double porosity as a twofold saddle point problem. The inf-sup condition in the discrete space takes the form

$$
\begin{aligned}
\sup _{\boldsymbol{v}^{h} \in \mathcal{S}_{u}^{h}} \frac{\int_{\Omega} B\left(\psi^{M} p_{M}^{h}+\psi^{m} p_{m}^{h}\right) \nabla \cdot \boldsymbol{v}^{h} \mathrm{~d} \Omega}{\left\|\boldsymbol{v}^{h}\right\|_{1}} & \geq C\left(\left\|B \psi^{M} p_{M}^{h}\right\|_{0}+\left\|B \psi^{m} p_{m}^{h}\right\|_{0}\right) \\
& \geq C\left(\left\|B\left(\psi^{M} p_{M}^{h}+\psi^{m} p_{m}^{h}\right)\right\|_{0}\right)
\end{aligned}
$$

for all $p_{M}^{h} \in \mathcal{S}_{p}^{h}$ and $p_{m}^{h} \in \mathcal{S}_{p}^{h}$, with $C>0$ independent of $h$.

Comparing (61) with (50), we find that the pressure constraint $p$ in singleconstraint problems is now replaced by the mean pore pressure weighted according to the pore fractions (and accommodating the Biot coefficient $B$ as well, to make the representation more physically meaningful):

$$
\bar{p}^{h}=B\left(\psi^{M} p_{M}^{h}+\psi^{m} p_{m}^{h}\right) .
$$

Note that both $p$ for single porosity and $\bar{p}$ for double porosity emanate from the effective stress equation. Following Bochev et al. [10], we now treat the twofold saddle point problem as a single saddle point problem in the mean pressures, and write the corresponding weaker condition as

$$
\sup _{\boldsymbol{v}^{h} \in \mathcal{S}_{u}^{h}} \frac{\int_{\Omega} \bar{p}^{h} \nabla \cdot \boldsymbol{v}^{h} \mathrm{~d} \Omega}{\left\|\boldsymbol{v}^{h}\right\|_{1}} \geq C_{1}\left\|\bar{p}^{h}\right\|_{0}-C_{2}\left\|\bar{p}^{h}-\Pi \bar{p}^{h}\right\|_{0}
$$

for all $p_{M}^{h} \in \mathcal{S}_{p}^{h}$ and $p_{m}^{h} \in \mathcal{S}_{p}^{h}$. Once again, the last term $C_{2}\left\|\bar{p}^{h}-\Pi \bar{p}^{h}\right\|_{0}$ quantifies the deficiency of the equal-order interpolations.

The stabilization term to penalize the resulting deficiency can be readily developed. The trial function for the stabilization term is $\bar{p}$ defined in (62). 
Accordingly, the Galerkin formulation dictates a similar form for the weighting function,

$$
\bar{\omega}^{h}=B\left(\psi^{M} \omega_{M}^{h}+\psi^{m} \omega_{m}^{h}\right) .
$$

This gives rise to the following stabilization term patterned after the expression proposed by White and Borja [90]:

$$
\begin{aligned}
\overline{\mathcal{M}}= & \frac{1}{\Delta t} \int_{\Omega} \frac{1}{2 G}\left(\bar{\omega}^{h}-\Pi \bar{\omega}^{h}\right)\left(\overline{\bar{p}^{h}-\Pi \bar{p}^{h}}\right) d \Omega \\
= & \frac{1}{\Delta t} \int_{\Omega} \frac{1}{2 G} B \psi^{M}\left(\omega_{M}^{h}-\Pi \omega_{M}^{h}\right)\left(\overline{\bar{p}^{h}-\Pi \bar{p}^{h}}\right) d \Omega \\
& +\frac{1}{\Delta t} \int_{\Omega} \frac{1}{2 G} B \psi^{m}\left(\omega_{m}^{h}-\Pi \omega_{m}^{h}\right)\left(\overline{\bar{p}^{h}-\Pi \bar{p}^{h}}\right) d \Omega .
\end{aligned}
$$

Observe that the expression for $\overline{\mathcal{M}}$ naturally splits into components associated with the two weighting functions for the macropore and micropore pressures. These components provide the necessary stabilization terms for the mass balance equations for the macropores and micropores. In terms of the residual vectors, we obtain (after multiplying $(65)$ by $\Delta t$ )

$$
\begin{aligned}
& \overline{\mathcal{R}}_{p_{M}}=\mathcal{R}_{p_{M}}+\int_{\Omega} \frac{1}{2 G} B \psi^{M}\left[\boldsymbol{N}^{p}-\Pi\left(\boldsymbol{N}^{p}\right)\right]\left[\left(\bar{p}^{h}-\bar{p}_{n}^{h}\right)-\Pi\left(\bar{p}^{h}-\bar{p}_{n}^{h}\right)\right] d \Omega, \\
& \overline{\mathcal{R}}_{p_{m}}=\mathcal{R}_{p_{m}}+\int_{\Omega} \frac{1}{2 G} B \psi^{m}\left[\boldsymbol{N}^{p}-\Pi\left(\boldsymbol{N}^{p}\right)\right]\left[\left(\bar{p}^{h}-\bar{p}_{n}^{h}\right)-\Pi\left(\bar{p}^{h}-\bar{p}_{n}^{h}\right)\right] d \Omega .
\end{aligned}
$$

Because the residual vectors $\overline{\mathcal{R}}_{p_{M}}$ and $\overline{\mathcal{R}}_{p_{m}}$ have been stabilized, the tangent operator also must be stabilized. The $2 \times 2$ lower right-hand submatrix block now contains the following additional terms

$$
\begin{aligned}
\bar{D} & =D+\int_{\Omega} \frac{1}{2 G}\left(B \psi^{M}\right)^{2}\left[\boldsymbol{N}^{p}-\Pi\left(\boldsymbol{N}^{p}\right)\right]\left[\boldsymbol{N}^{p}-\Pi\left(\boldsymbol{N}^{p}\right)\right] d \Omega \\
\bar{E}_{1} & =E_{1}+\int_{\Omega} \frac{1}{2 G}\left(B^{2} \psi^{M} \psi^{m}\right)\left[\boldsymbol{N}^{p}-\Pi\left(\boldsymbol{N}^{p}\right)\right]\left[\boldsymbol{N}^{p}-\Pi\left(\boldsymbol{N}^{p}\right)\right] d \Omega \\
\bar{E}_{2} & =E_{2}+\int_{\Omega} \frac{1}{2 G}\left(B^{2} \psi^{m} \psi^{M}\right)\left[\boldsymbol{N}^{p}-\Pi\left(\boldsymbol{N}^{p}\right)\right]\left[\boldsymbol{N}^{p}-\Pi\left(\boldsymbol{N}^{p}\right)\right] d \Omega \\
\bar{F} & =F+\int_{\Omega} \frac{1}{2 G}\left(B \psi^{m}\right)^{2}\left[\boldsymbol{N}^{p}-\Pi\left(\boldsymbol{N}^{p}\right)\right]\left[\boldsymbol{N}^{p}-\Pi\left(\boldsymbol{N}^{p}\right)\right] d \Omega
\end{aligned}
$$

Consequently the new tangent operator now takes the form

$$
\left[\begin{array}{ccc}
A & B_{1} & C_{1} \\
B_{2} & D & E_{1} \\
C_{2} & E_{2} & F
\end{array}\right] \rightarrow\left[\begin{array}{ccc}
A & B_{1} & C_{1} \\
B_{2} & \bar{D} & \bar{E}_{1} \\
C_{2} & \bar{E}_{2} & \bar{F}
\end{array}\right]
$$


Note that the additional stabilization terms form a symmetric matrix as a result of the Galerkin formulation.

Before closing this section, we briefly address the issue of solvability of the undrained problem with double porosity. The extreme case of interest occurs when the matrices $\bar{D}, \bar{E}_{1}, \bar{E}_{2}$, and $\bar{F}$ are all zero, which corresponds to fully undrained condition at the two pore scales and without stabilization. Ignoring the mass transfer terms, the coefficient matrix becomes symmetric when $A=A^{\top}$, i.e.,

$$
\left[\begin{array}{ccc}
A & B_{1} & C_{1} \\
B_{2} & \bar{D} & \bar{E}_{1} \\
C_{2} & \bar{E}_{2} & \bar{F}
\end{array}\right] \rightarrow\left[\begin{array}{ccc}
A & B_{2}^{\top} & C_{2}^{\top} \\
B_{2} & 0 & 0 \\
C_{2} & 0 & 0
\end{array}\right] \rightarrow\left[\begin{array}{cc}
A & G^{\top} \\
G & 0
\end{array}\right],
$$

where $G^{\top}=\left[\begin{array}{ll}B_{2}^{\top} & C_{2}^{\top}\end{array}\right]$. We see that undrained loading in a double porosity medium creates a condition in which the width of the coupling matrix $G^{\top}$ increases about two times relative to that emanating from a single porosity problem. This means that the null submatrix block on the diagonal also doubles in size relative to the single porosity problem.

The solvability aspects of a problem having a coefficient matrix of the form given by (73) have been addressed by Brezzi and Bathe [18], who in turn credited Arnold [4] for the 'elegant' formulation. In a nutshell, the undrained problem is solvable provided that: (a) the size of $A$ is greater than the size of the null submatrix block on the diagonal, thus ensuring that the kernel set of $G$ is not an empty set; (b) the square matrix formed from $G$ by eliminating the elements of the kernel space of $G$ is not singular, and (c) the square submatrix of $A$ associated with the kernel space of $G$ is not singular. We also refer to $[37,43]$ for further discussions on the solvability of the discrete system of the form given by (73).

\section{Numerical examples}

This section presents numerical examples demonstrating the performance and efficacy of the proposed stabilized mixed finite elements. We begin by verifying our finite elements through Cryer's sphere, a classic single porosity poromechanics problem for which an analytical solution exists. Subsequently, we perform a comparative investigation into the stabilized low-order mixed finite elements with intrinsically stable high-order and unstable low-order elements. For this purpose, we first consider an undrained double porosity sphere under spatially varying loading. We then introduce a plane strain strip footing problem and examine the efficacy of the proposed stabilization scheme when the permeabilities of the two pore scales are orders of magnitude different. This difference not only affects the pressure difference between the macropores and micropores, but also controls the combinations of drainage regimes in the two scales. 
The numerical analyses have been conducted using Geocentric, a massively parallel finite element code for geomechanics that is built upon the deal.II finite element library [7,8], p4est mesh handling library [20], and the Trilinos project [42]. We more or less followed the standard recipes of those libraries except the solver. Due to a large size of the linear system stemming from a three-field mixed formulation, we implemented an iterative solver aided by a block-preconditioner that exploits the $3 \times 3$ block-partitioned structure and spectral properties of the tangent operator. In essence, the solution strategy is an extended version of the block preconditioned Newton-Krylov solver for single porosity proposed in [91].

The following notations are used for referring to the mixed finite elements of interest. The interpolation for displacement is denoted by $\mathrm{Q}$ and for pressure by $\mathrm{P}$, followed by a number representing the order of interpolation. For example, Q2P1P1 is a mixed element employing second-order (quadratic Lagrangian) interpolation for displacement and first-order (linear) interpolation for the two pressures, while Q1P1P1 adopts the same linear interpolation for displacement and the two pressure fields. We also use a lower case "s" to denote stabilization, i.e., Q1P1P1s refers to the stabilized mixed finite elements investigated in this paper. Throughout this section we have employed the same level of interpolation for the two pressure fields, and used the same pressure nodes for the micropore and macropore pressure interpolations.

\subsection{Cryer's sphere}

The objective of our first example is to verify the formulation and implementation of our mixed finite elements for double porosity poromechanics. We are not aware of a closed-form analytical solution to any problem related to fully coupled double porosity poromechanics, so we have selected the Cryer's sphere problem [26] as a test example. Cryer's sphere is a classic single porosity problem that can be solved by Laplace transform. In this problem a saturated poroelastic sphere is compressed by applying a uniform pressure on its outer surface. The analytical solution of this problem indicates that the excess pore pressure initially rises before it begins to dissipate, a phenomenon often referred to in the literature as the Mandel-Cryer effect. This initial pressure increase is a signature feature of coupled responses between solid deformation and fluid diffusion. The Cryer's sphere problem has served as a useful reference in the past to verify various formulations and implementation of coupled poromechanics solutions.

For verification we performed single porosity simulations of the Cryer sphere problem with the proposed double porosity mixed finite elements. This was done by assigning a null porosity to all integration points of the micropores, and zero micropore pressures for the initial and boundary conditions at the nodes. Mass transfer was ignored as well. By comparing results from single porosity finite elements, we confirmed that this setting successfully emulates a single porosity condition by maintaining zero pressures in the micropores 
throughout all time steps. We also checked to verify that the double porosity mixed elements could capture the analytical solution by suppressing the macropore pressures and activating only the micropore pressure degrees of freedom.

We conducted the simulations using three types of mixed finite elements: Q2P1P1, Q1P1P1, and Q1P1P1s. We modeled an octant of a unit sphere taking advantage of symmetry. For Q2P1P1 the domain was discretized with 3456 quadrilateral elements, resulting in a total of 98245 degrees of freedom (90099 for displacement and 4073 for each pressure type); for Q1P1P1s the same number of elements resulted in a total of 20365 degrees of freedom (12219 for displacement and 4073 for each pressure type). Note that the total number of degrees of freedom generated by Q2P1P1 elements is much higher than that generated by the lower-order elements. In addition, higher-order elements also require a greater number of integration points. The potentially significant reduction in computing effort is the primary motivating factor for stabilizing these elements.

The solution of Cryer's sphere problem is a function of Poisson's ratio for the solid matrix. Therefore, we considered three values of Poisson's ratio: $\nu=0,0.25$ and 0.4 , and fixed all other parameters at constant values except for the permeability coefficient that was adjusted to normalize the time axis. The rest of the parameters were assigned the following values: bulk modulus $K=1000 \mathrm{kPa}, \rho_{s}=\rho_{f}=1.0 \mathrm{t} / \mathrm{m}^{3}, \phi^{M}=0.5$ or $\phi^{m}=0.5$ with $\phi^{M}+\phi^{m}=0.5$, $\mu_{f}=10^{-6} \mathrm{kPa} \cdot \mathrm{s}$, and $k_{f}=3.33 \times 10^{-12}$ to $8.18 \times 10^{-12} \mathrm{~m}^{2}$. We assumed $B=1$, which is a realistic value for soils. We simulated the sphere problem until the non-dimensionalized time factor $T=c_{v} t / r^{2}$ has reached a value of 0.2 after using 40 time steps, where $c_{v}$ is the coefficient of consolidation, $t$ is time, and $r$ is the drainage length. In what follows, we express the solutions in terms of normalized pore pressure $p / p_{0}$, where $p$ is the pore pressure normalized with respect to the boundary pressure $p_{0}$.

Figure 1 shows the distribution of normalized pore pressure $p / p_{0}$ at the initial time step $T=0.005$ when $\nu=0$. The three types of element gave virtually the same results, so we only present the solutions from the Q1P1P1simulations in this figure. Also, the double porosity simulations obtained by activating either only the macropores or only the micropores gave identical solutions; therefore, the figure does not distinguish between the two solutions. We find that even without stabilization equal-order linear interpolations do not exhibit pressure oscillations, since this problem deals with drained conditions right from the initial time step (note that the focus of this example is only on the verification of the finite element implementation, and not on stabilization during undrained condition). In Figure 2 we show the evolutions of $p / p_{0}$ at the center of the sphere predicted by the analytical and numerical solutions. The three mixed elements provided essentially the same results that agree with the analytical solutions. These results thus verify the formulation and implementation of the mixed finite elements under drained loading conditions. 


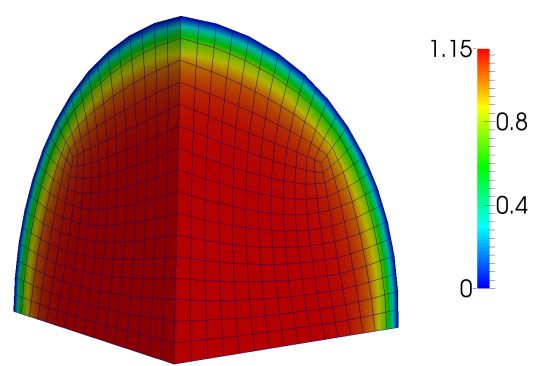

Fig. 1. Normalized pore pressure $p / p_{0}$ in Cryer's sphere with $\nu=0$ at time factor $T=0.005$.

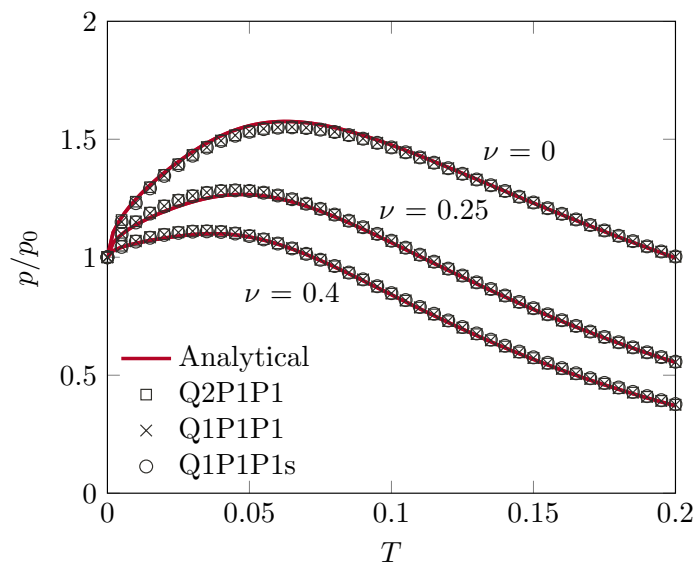

Fig. 2. Comparison of analytical and numerical solutions at the center of Cryer's sphere.

We note two important observations from this example. First, under drained condition the equal-order unstabilized element, Q1P1P1, performs well and does not exhibit any instability. Second, the PPP technique does not introduce any numerical artifacts that significantly alter the drained solution. This latter feature is important because, as noted earlier, poromechanics problems typically cover the entire range of drainage conditions, from fully undrained to fully drained. Therefore, a robust stabilization scheme should not alter the solution in the regime where no stabilization is needed. The next example shows how the proposed stabilization scheme can improve the performance of an inherently unstable mixed element in the undrained regime.

\subsection{Undrained double porosity sphere}

Having verified our mixed finite elements, we now turn our attention to undrained deformation in double porosity material and consider the same 
unit sphere as a next example. In this example, all boundaries of the sphere were treated as no-flux boundaries. The modified problem does not allow an analytical solution, but it does provide a simple setting for investigating the efficacy of the proposed stabilization technique in a 3D double porosity problem.

We assumed a double porosity material with $\phi^{M}=0.3$ and $k_{M}=6 \times$ $10^{-14} \mathrm{~m}^{2}$ for the macropores, and $\phi^{m}=0.1$ and $k_{m}=6 \times 10^{-17} \mathrm{~m}^{2}$ for the micropores, resulting in a permeability ratio of $k_{M} / k_{m}=10^{3}$. The two pore scales were coupled with mass transfer terms in which $a=0.1 \mathrm{~m}, \beta=3.0$, and $\gamma=0.4$. For the solid matrix we assigned $K=1000 \mathrm{kPa}$ and $\nu=0.25$. All other parameters remain the same as in the previous example. To impose pressure variations in the domain, we applied a spatially varying loading $1+$ $0.5 \sin (x) \mathrm{kPa}$ on its outer surface. This pressure variation perturbed the stress field from the isotropic condition considered by Cryer to a full 3D stress field within the sphere.

Figure 3 compares the excess pore pressures obtained using the three mixed elements after 0.1 seconds of loading. From the results of simulations with Q2P1P1 elements, we first observe that pressure distributions in the micropores are more localized than those in the macropores due to the much lower permeability of the micropores. The micropore pressures predicted by the unstabilized Q1P1P1 elements exhibit a checkerboard pattern, which is a typical spurious mode associated with numerical instability. We also observe some mild macropore pressure oscillations produced by the unstabilized Q1P1P1 elements in places with the pressure gradients are high. In contrast, the reference solution provided by the higher-order Q2P1P1 elements is smooth. Furthermore, the results obtained from the stabilized Q1P1P1s elements also show a smooth pattern that is nearly identical to those produced by the higher-order Q2P1P1 elements. We note that the stabilization successfully eliminated oscillation at the two scales, resulting in nearly identical solutions to those provided by the higher-order elements.

\subsection{Strip footing}

Our next example is a plane strain strip footing problem on a foundation material with double porosity. We adopted a typical configuration of strip footing for this example. Figure 4 illustrates the specific geometry and boundary conditions. Considering symmetry, we modeled only one-half of a $4 \mathrm{~m}$ wide footing resting on the ground. The fluid boundary conditions were identical for both pore scales except for the ground surface, which was assumed to be a homogeneous Dirichlet boundary for the macropores (i.e., $p_{M}=0$ ) and a Neumann boundary (no flux) for the micropores.

The reason for suppressing drainage through the micropore ground surface is to eliminate unnecessary oscillations occurring near the drainage boundary of a low-permeability medium (see [66] for example). This oscillation is due to 
Macropores
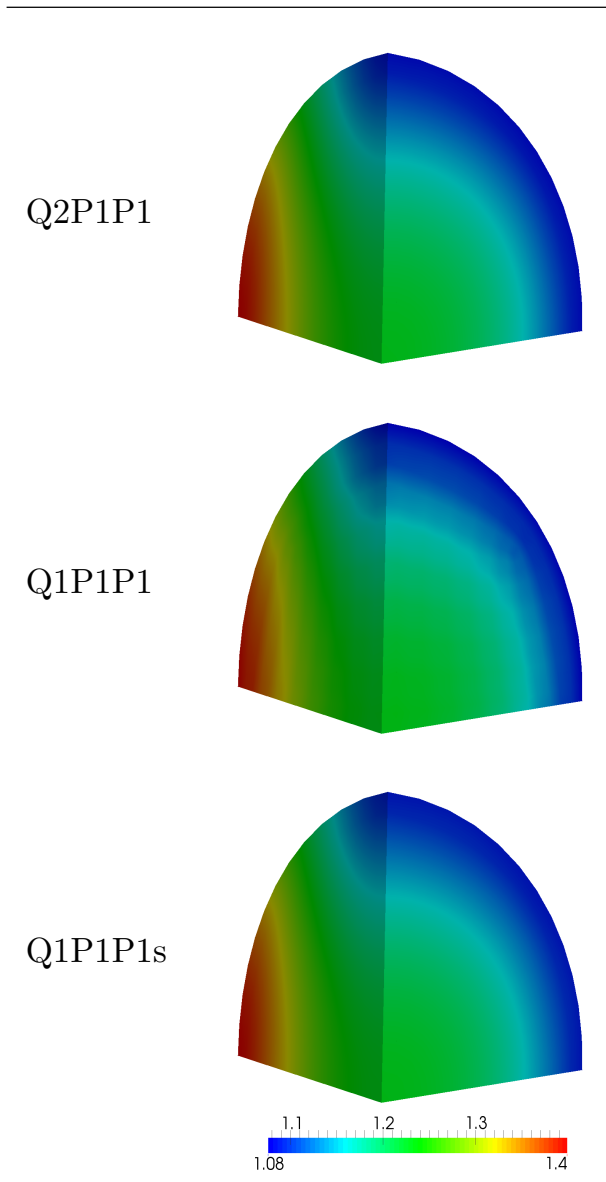

Micropores
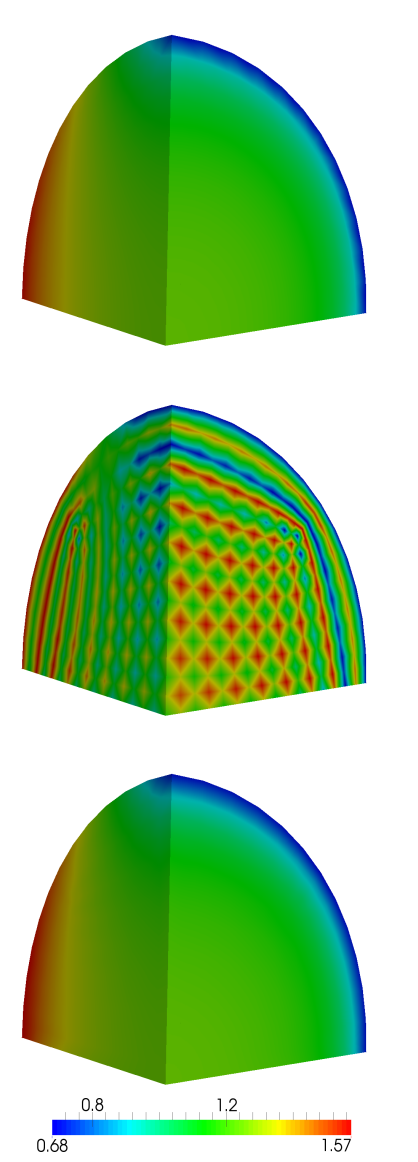

Fig. 3. Excess pore pressures in double porosity sphere after 0.1 seconds of undrained loading. Surface pressure varies spatially according to $1+0.5 \sin (x)$.

sharp pressure gradients, or shocks, which is not related to the spurious pressure oscillation stemming from the inf-sup instability. It has been shown (see Appendix A of [52]) that shocks in the form of sharp pressure gradients can be treated by other techniques, such as the finite volume method. Stabilization of such oscillation near drainage boundaries is a topic of other studies (see e.g., [71]) and will not be covered in this work. To fully focus on the instability at hand, we assumed the micropore scale as a globally undrained medium. Regarding the displacement field, we fixed the vertical displacements at the bottom boundary and the horizontal displacements at the two vertical sides. We selected point A, which is located $0.5 \mathrm{~m}$ below the center of the footing, 
as a reference point for reporting the evolutions of the two pore pressures and displacement with time.

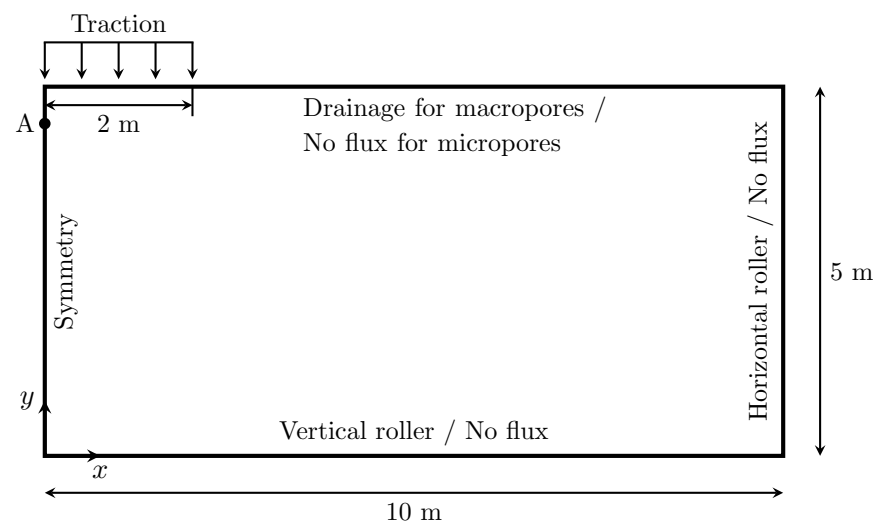

Fig. 4. Schematic illustration for the geometry and boundary conditions of the strip footing problem.

We investigated the mesh sensitivity issue by employing two finite element meshes depicted in Fig. 5. The total number of degrees of freedom with Q2P1P1 and Q1P1P1(s) mixed elements are 8364 and 3444 for Mesh \#1, and 32724 and 13284 for Mesh \#2, respectively. We note that even in 2D analysis, the reduction in the total number of degrees of freedom engendered by the low-order stabilized elements is still fairly significant, although it is not as significant in this case as in the 3D problem of the previous example.

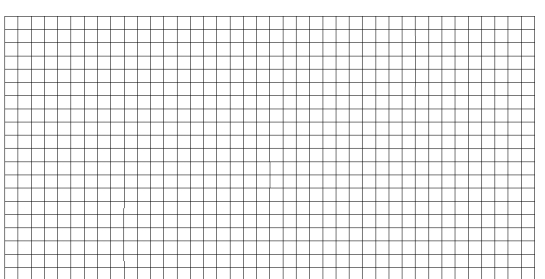

Mesh \#1

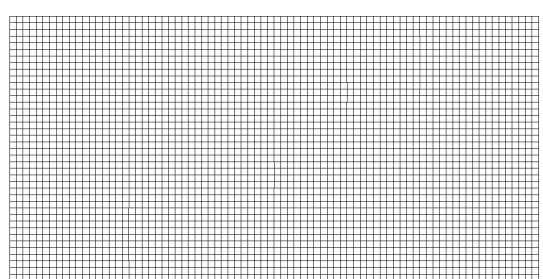

Mesh \#2

Fig. 5. Two meshes used in the strip footing example. Mesh \#1 has 800 elements while Mesh \#2 has 3200 elements.

We examined the performance of the proposed stabilization scheme by considering the permeabilities of the macropores and micropores $\left(k^{M}\right.$ and $k_{m}$, respectively) to be several orders of magnitude different. For the micropores, we assumed the following values: $\phi^{m}=0.1$ and $k_{m}=5 \times 10^{-17} \mathrm{~m}^{2}$. For the 
macropores, we assumed $\phi^{M}=0.05$ and $k_{M}=1 \times 10^{-10} \mathrm{~m}^{2}$ for Case \#1, and decreased the permeability to $5 \times 10^{-15} \mathrm{~m}^{2}$ for Case \#2. This leads to a drop in the permeability ratio $k_{M} / k_{m}$ from a value of $2 \times 10^{6}$ for Case \#1 to a value of $1 \times 10^{2}$ for Case \#2. As will be shown later, this difference in the permeability ratio also alters the combination of drainage conditions from the initial step. The remaining parameters were identical for both cases.

We activated the diffusive mass transfer between two pore scales, with $a=5 \times 10^{-2} \mathrm{~m}, \gamma=0.4$ and $\beta=11.0$. We assumed the following properties for the pore fluid: $\rho_{f}=1.0 \mathrm{t} / \mathrm{m}^{3}$ and $\mu_{f}=1 \times 10^{-6} \mathrm{kPa} \cdot \mathrm{s}$; and for the solid matrix: $\rho_{s}=2.6 \mathrm{t} / \mathrm{m}^{3}$ and $B=0.9$. We considered the foundation medium to be linearly elastic with bulk modulus $K=2000 \mathrm{kPa}$ and Poisson's ratio $\nu=0.2$. We applied the footing load as a ramp function that increases from an initial value of zero to a final value $20 \mathrm{kPa}$ over a period of $180 \mathrm{~s}$, after which it was held constant at $20 \mathrm{kPa}$ for the next $180 \mathrm{~s}$. Time steps taken were $\Delta t=5 \mathrm{~s}$ during the ramp-loading phase, and $\Delta t=10 \mathrm{~s}$ thereafter.

In what follows, we consider the variation and distribution of the excess pore pressure fields induced by the footing load. We first focus on the two pore pressure fields at the initial time step when the system is deforming in undrained fashion within the entire time scale. In particular, we focus on the pressure variations below the center of the footing (i.e., along the symmetry line). We then present time histories of macropore and micropore pressures at Point A illustrated in Fig. 5.

\section{Case \#1: Higher permeability contrast}

We first consider the case in which the macropores are $2 \times 10^{6}$ times more permeable than the micropores. This condition is analogous to that of a highly fissured rock, where the permeability of the fissures is several orders of magnitude higher than that of the rock matrix. Figure 6 compares the macropore and micropore pressures below the center of the footing at the initial step. Significant difference can be observed between the two pore pressure fields both quantitatively and qualitatively. Due to the much lower drainage within the time interval, the micropore pressures are around two orders of magnitude higher than the macropore pressures. Also, the micropore pressures decrease from the top drainage surface to the bottom, while the macropore pressures slightly increase with depth.

Setting the foregoing results as the reference solution, we next focus on the stability of the Q1P1P1 mixed elements with Mesh \#1. The macropore pressures calculated with these unstable elements are nearly the same as those calculated with Q2P1P1 elements. However, the micropore pressures exhibit pronounced spurious oscillations. With Mesh \#2, on the other hand, these oscillations are somewhat suppressed but not completely eliminated. In contrast, the stabilized Q1P1P1s elements provide smooth solutions that are nearly identical to those obtained with the Q2P1P1 elements. As the mesh 
is refined (Mesh \#2), the stabilized solutions became even closer to those calculated by the higher-order elements.
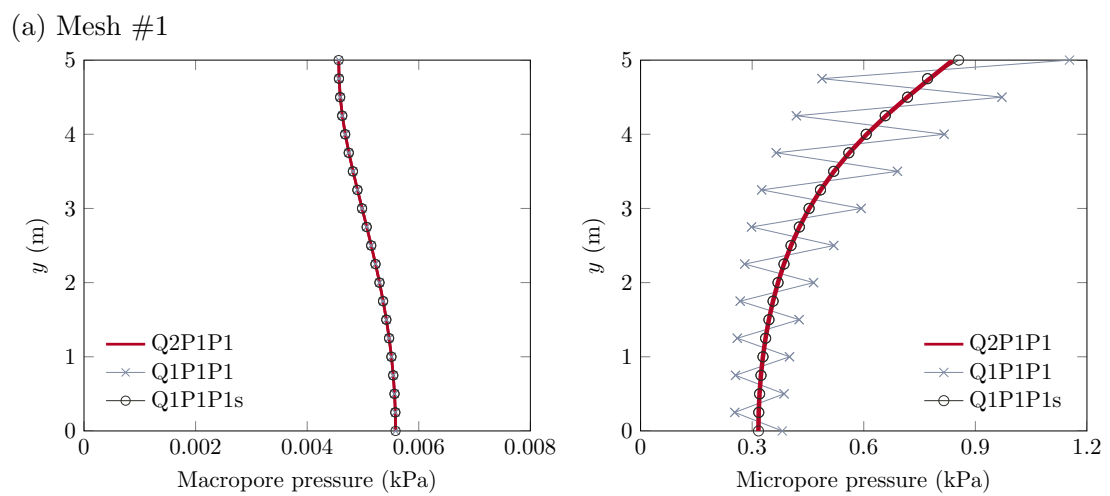

(b) Mesh \#2
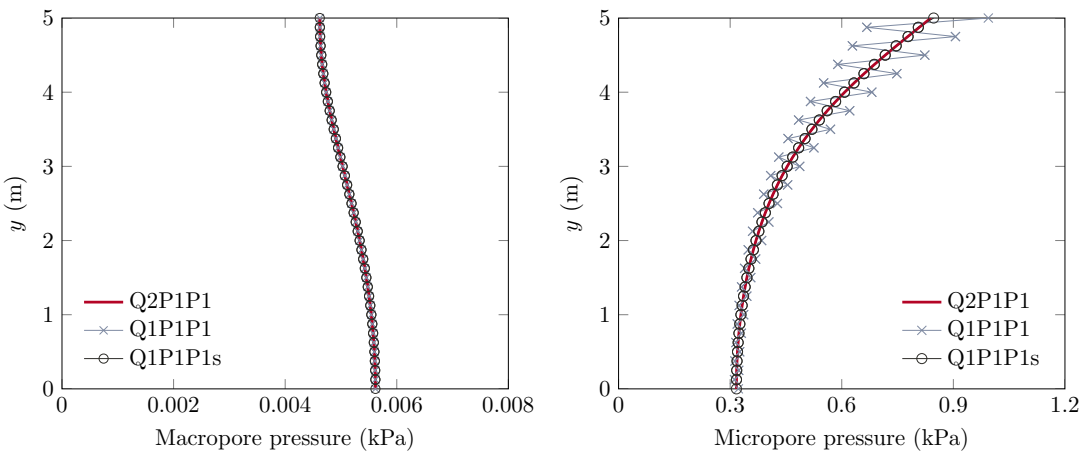

Fig. 6. Pore pressure variations below the center of footing at the initial time step: Case \#1 $\left(k_{M} / k_{m}=2 \times 10^{6}\right)$.

In Fig. 7 we compare the two pore pressure fields generated at the same time instant using Mesh \#2. As expected, the two pore pressure fields show completely different distributions throughout the entire domain. With respect to stability, the unstabilized equal-order pair Q1P1P1 elements exhibit spurious micropore pressure oscillations, although the macropore pressure distribution shows a smooth, stable pattern. These features are unique to double porosity formulation and is not encountered in single porosity poromechanics, i.e., stability on one scale does not necessarily imply stability on another scale. More importantly, the proposed stabilization technique completely circumvents instabilities irrespective of scale - it suppresses instabilities where they would otherwise arise, and preserves the "correct" response at the scale where no stabilization is needed. 


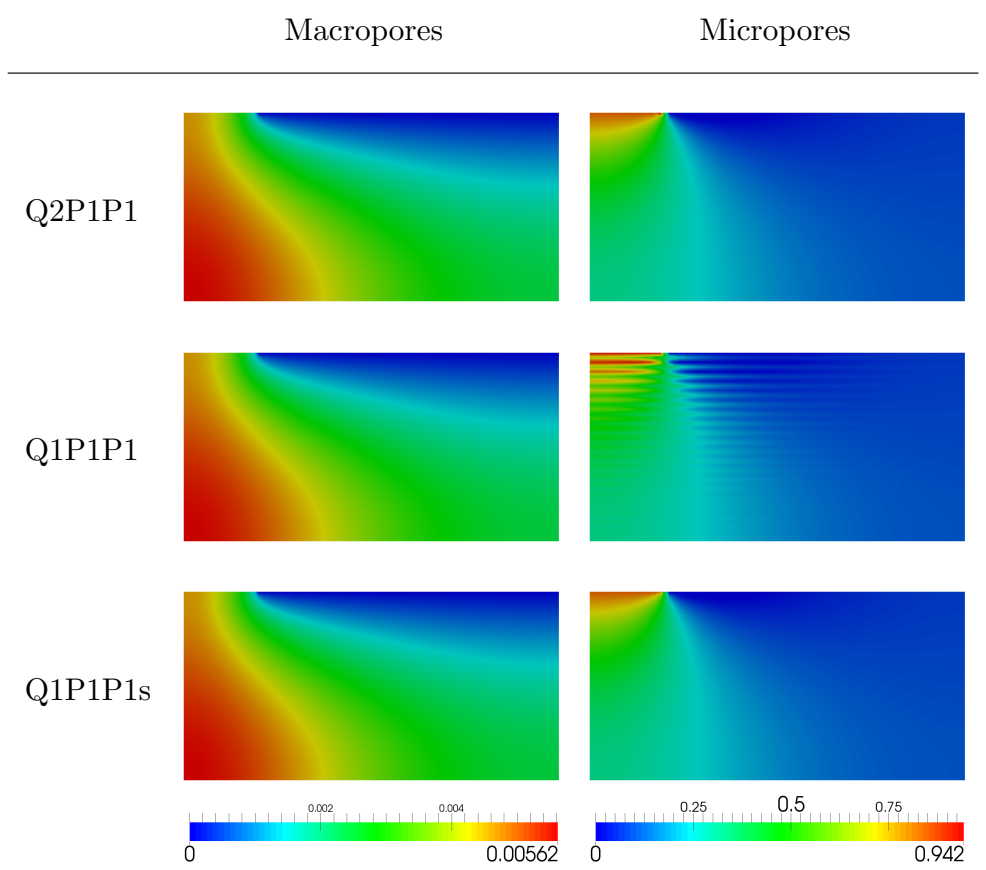

Fig. 7. Contours of macropore and micropore pressures at the initial time step: Case \#1 $\left(k_{M} / k_{m}=2 \times 10^{6}\right)$ with Mesh \#2.

We next examine the time-evolution of pore pressures at Point A shown in Fig. 8. Not surprisingly, all three mixed elements yield essentially the same macropore pressure-time responses. The micropore pressure response calculated by the unstabilized Q1P1P1 elements still deviates slightly from that calculated by the higher-order elements, but the deviation is not significant. No oscillation in time domain is observed, a testament of the high-frequency numerical damping of the backward Euler method. Note that both the macropore and micropore pressure histories show a decreasing trend during the constant footing load period: the macropore pressure decreases as fluid drains into the ground surface, while the micropore pressure decreases as the micropore fluids drain into the macropores (recall that the micropore fluids cannot drain into the exterior boundaries because of the no-flux boundary condition on all exterior surfaces prescribed for the micropore scale). On the other hand, Figure 9 shows that the displacement at Point A remains nearly unaffected by the pressure instabilities.

\section{Case \#2: Lower permeability contrast}

Next we investigate the instability patterns and examine the performance of the proposed stabilization scheme when the ratio of the two permeabilities is 
(a) Mesh \#1
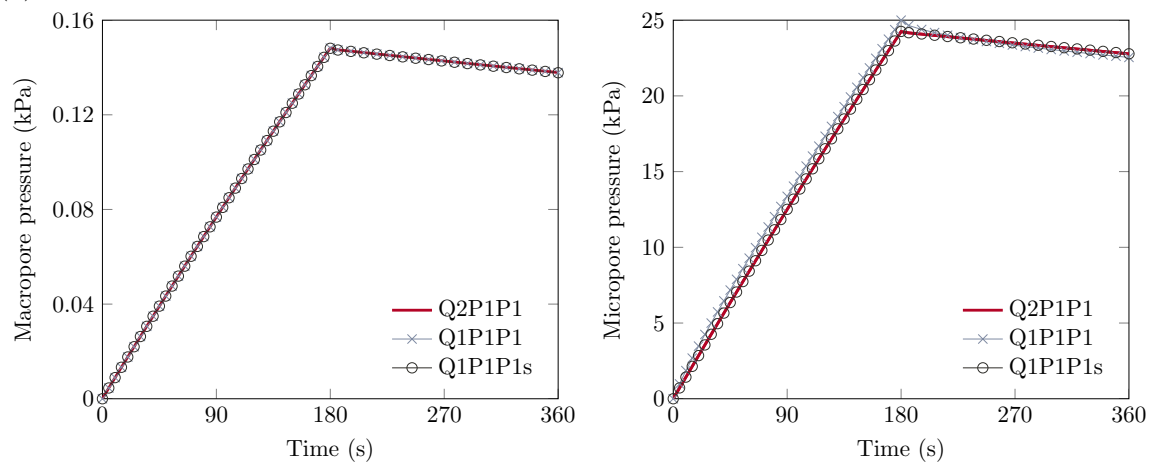

(b) Mesh \#2
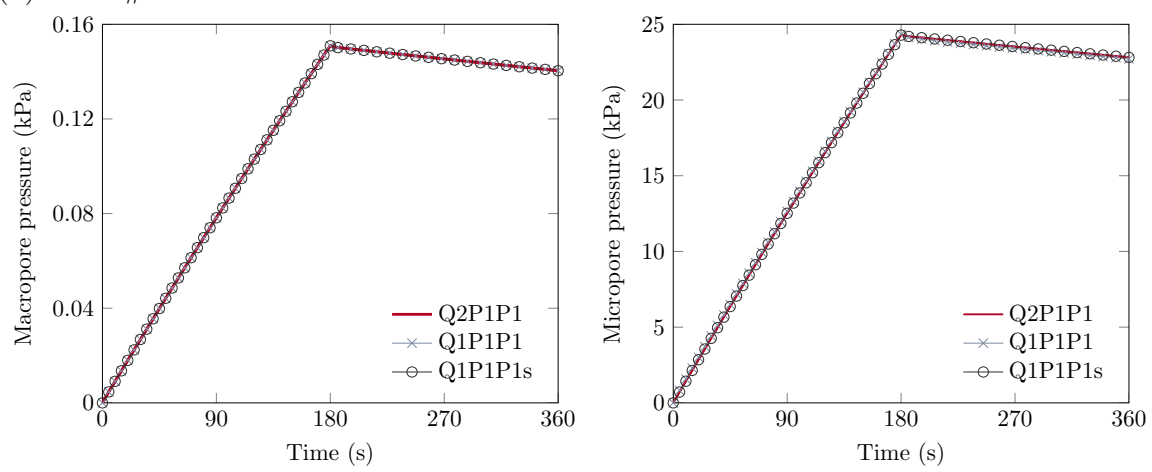

Fig. 8. Time histories of pore pressures at Point A: Case \#1 $\left(k_{M} / k_{m}=2 \times 10^{6}\right)$.

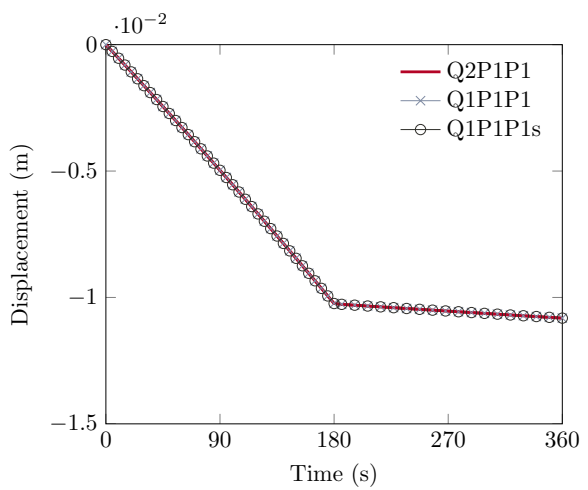

Fig. 9. Time history of displacement at Point A: Case \#1 $\left(k_{M} / k_{m}=2 \times 10^{6}\right)$ with Mesh \#1. 
lower, at $k_{M} / k_{m}=1 \times 10^{2}$. Figure 10 shows the pore pressure variations below the center of the footing at the initial time step. We see that the macropore and micropore pressures calculated by the Q2P1P1 elements are now fairly close to each other, unlike in the previous simulations where they were orders of magnitude different. This is attributed to extensive diffusive mass transfer that allowed the two pore pressures to be nearly the same.

Figure 10 shows that simulations with Q1P1P1 elements along with the coarser Mesh \#1 now exhibit spurious oscillations in both the macropore and micropore pressures. The oscillations have been somewhat alleviated with the finer Mesh \#2, but not completely circumvented particularly on the micropore scale. However, the proposed stabilization scheme completely eliminates these oscillations at both scales, suggesting the efficacy of the technique when simultaneous multiscale stabilization is required. Furthermore, the results show that instabilities do not depend on the magnitude of the pressure per se, but rather, on the degree of permeability of the material. This can be gleaned from the fact that the calculated macropore and micropore pressures are nearly the same for this example, yet the magnitudes of oscillation are significantly different.

Figure 11 shows the overall distributions of the macropore and micropore pressures at the initial time step obtained from the finer Mesh \#2. Qualitatively, the pore pressure distributions within the two scales are somewhat similar, although the micropore pressure distribution is a little bit more concentrated below the footing. The unstabilized Q1P1P1 elements once again exhibit pore pressure oscillations at both scales, although oscillations at the macropore scale concentrate more in places with sharp pressure gradients, and are generally milder. In contrast, the stabilized Q1P1P1s elements generate smooth pressure distributions throughout, and essentially reproduce the results generated by the higher-order Q2P1P1 elements,

Figure 12 shows the time histories of the macropore and micropore pressures at the reference point $\mathrm{A}$. We see that the Q1P1P1 solutions with Mesh \#1 generated larger numerical errors in the micropore pressures, although errors in the macropore pressures are much smaller. Even though the permeability of the micropore region is the same as in the first case considered, the errors in time histories become much larger in the present case. This can be explained by extending the reasoning provided in the previous case: since the permeability contrast is much lower in the present case, fluids in the micropores cannot drain as easily into the macropores, because the permeability of the macropores is now closer to that of the micropores. The virtually constant post-peak pressure, which was decreasing in the previous case, is evidence of this drainage difference. However, these errors seem to vanish when the finer Mesh \#2 is used. Also, in all cases, the time histories of Q2P1P1 and Q1P1P1s are nearly the same, suggesting that stabilization is indeed effective. While not presented in this paper, we also point out that the spurious oscillations observed in the pore pressure fields has not impacted the stability of the displacement field. 
(a) Mesh \#1
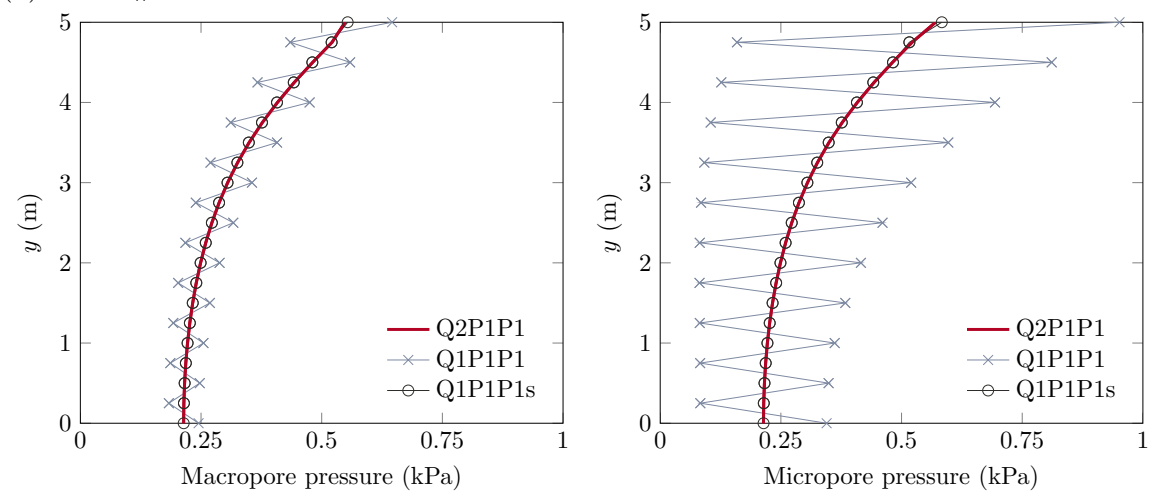

(b) Mesh \#2
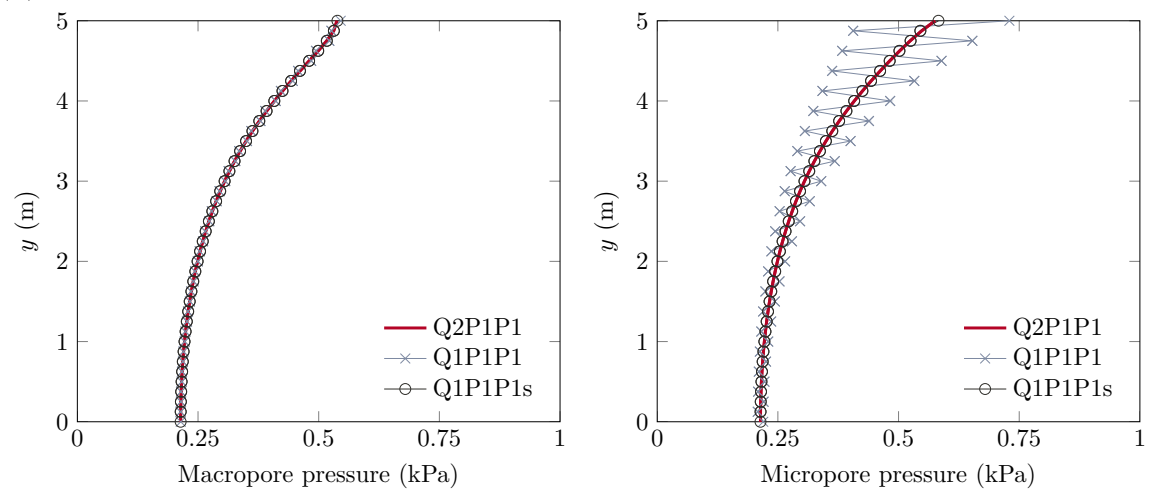

Fig. 10. Pore pressure variations below the center of footing at the initial time step: Case \#2 $\left(k_{M} / k_{m}=1 \times 10^{2}\right)$.

\section{Closure}

We have presented stabilized low-order mixed finite elements for coupled solid deformation and fluid diffusion in a material exhibiting two pore scales. The stabilization is a variant of the PPP technique developed for dual treatment of two distinct pore pressure constraints. One appealing aspect of the proposed technique is the physical motivation behind the stabilization: the mean trial pore pressure is a weighted mean determined according to the pore fractions, and so the mean weighting function should also be a weighted mean of the pore pressure variations determined according to the same pore fractions. Remarkably, this physical motivation is backed up by mathematical developments, in which a twofold saddle point problem is used to develop an equivalent single saddle point problem in terms of the weighted mean pore pressures. This leads to a parameter-free PPP stabilization technique for the equivalent 


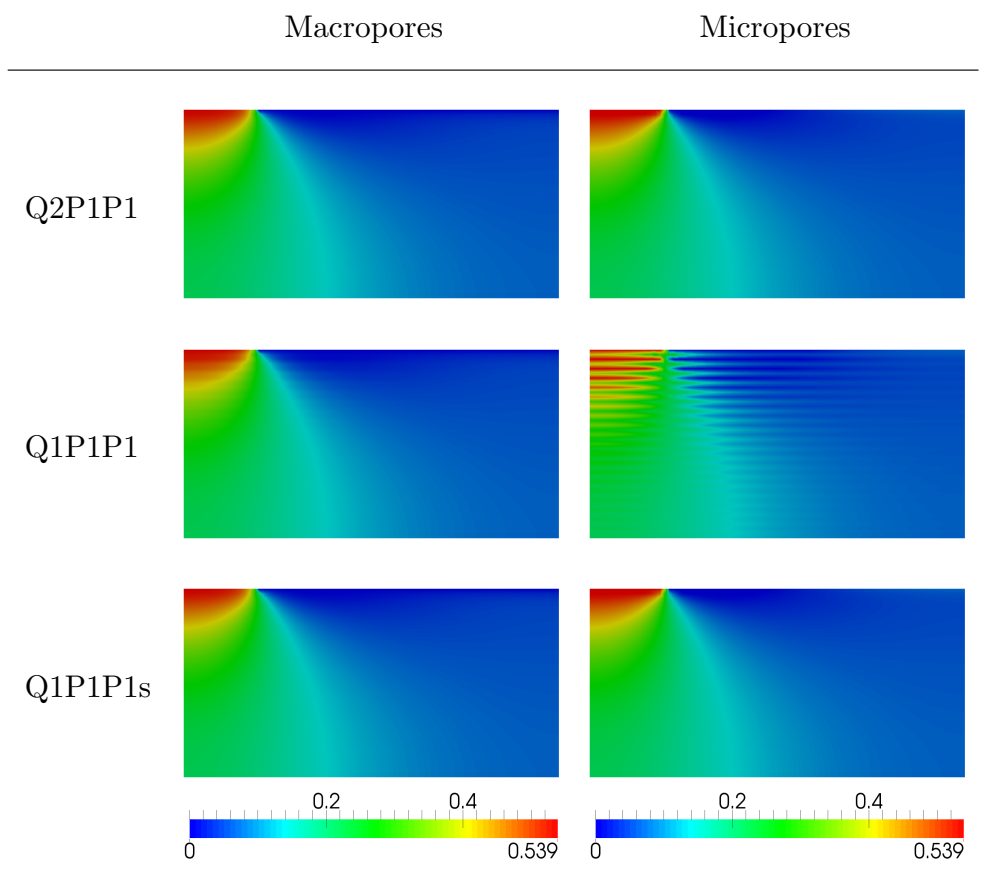

Fig. 11. Distributions of macropore and micropore pressures at the initial time step: Case \#2 $\left(k_{M} / k_{m}=2 \times 10^{2}\right)$ with Mesh \#2.

single saddle point problem in the mean pore pressures, which allows equalorder interpolations of the solid displacement and the two pore pressure fields. Numerical examples have demonstrated the efficacy of the proposed stabilization technique under various drainage conditions, from fully undrained to fully drained conditions, and from 2D to 3D loading and drainage configurations. Results of these studies can have a significant impact on the stabilization of numerical algorithms related to similar multiphysical and multiscale problems in science and engineering, such as those arising in contact problems.

\section{Acknowledgements}

This material is based upon work supported by the U.S. Department of Energy, Office of Science, Office of Basic Energy Sciences, Geosciences Research Program, under Award Number DE-FG02-03ER15454. The authors are grateful to Dr. Joshua A. White for his valuable assistance in the finite element implementation of the proposed algorithm. The first author acknowledges financial supports provided by the Fulbright Program and the John A. Blume Earthquake Engineering Center. 
(a) Mesh \#1

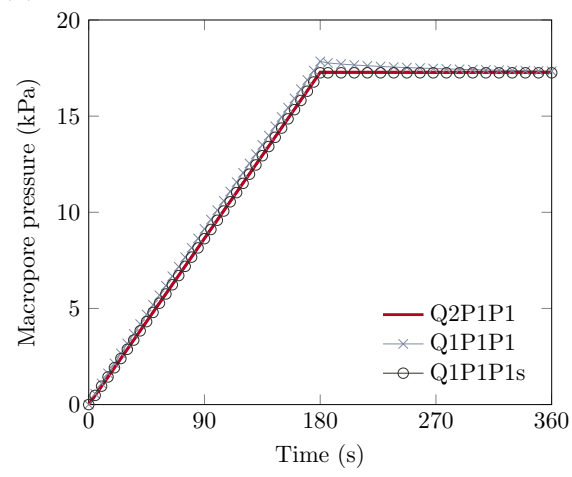

(b) Mesh \#2

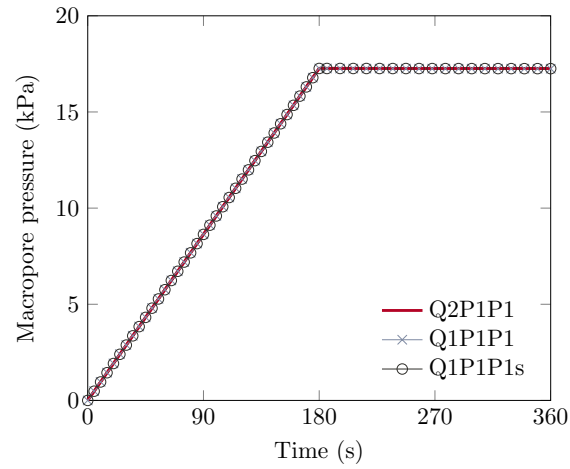

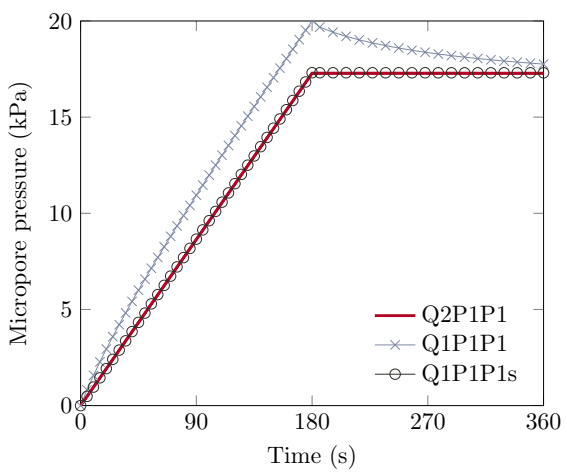

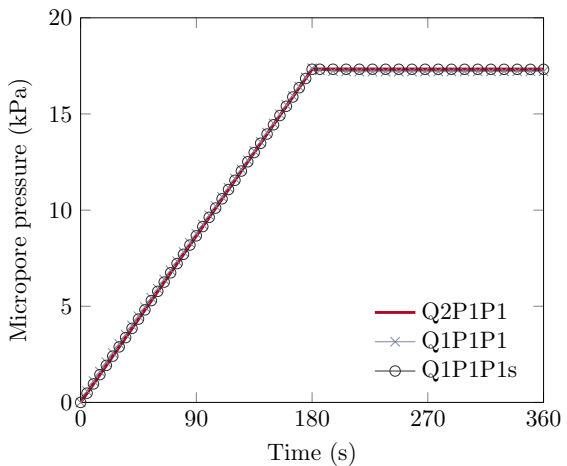

Fig. 12. Time histories of pore pressures at Point A: Case $\# 2\left(k_{M} / k_{m}=1 \times 10^{2}\right)$.

\section{References}

[1] Y. N. Abousleiman, S. K. Hoang, and C. Liu. Anisotropic porothermoelastic solution and hydro-thermal effects on fracture width in hydraulic fracturing. International Journal for Numerical and Analytical Methods in Geomechanics, 38(5):493-517, 2014.

[2] J. E. Andrade and R. I. Borja. Capturing strain localization in dense sands with random density. International Journal for Numerical Methods in Engineering, 67(11):1531-1564, 2006.

[3] T. Arbogast, J. Douglas Jim, and U. Hornung. Derivation of the double porosity model of single phase flow via homogenization theory. SIAM Journal on Mathematical Analysis, 21(4):823-836, 1990.

[4] D. Arnold. Discretization by finite elements of a model parameter dependent problem. Numerische Mathematik, 37:405-421, 1981.

[5] I. Babuška. The finite element method with Lagrangian multipliers. Numerische Mathematik, 20:179-192, 1973. 
[6] M. Bai, Q. Ma, and J. Roegiers. Dual-porosity behaviour of naturally fractured reservoirs. International Journal for Numerical and Analytical Methods in Geomechanics, 18:359-376, 1994.

[7] W. Bangerth, R. Hartmann, and G. Kanschat. deal. II-a general-purpose object-oriented finite element library. ACM Transactions on Mathematical Software, 33(4), 2007.

[8] W. Bangerth, T. Heister, L. Heltai, G. Kanschat, M. Kronbichler, M. Maier, B. Turcksin, and T. D. Young. The deal. II Library, Version 8.1. arXiv preprint, 1312.2266:1-5, 2013.

[9] G. Barenblatt, I. Zheltov, and I. Kochina. Basic concepts in the theory of seepage of homogeneous liquids in fissured rocks. Journal of Applied Mathematics and Mechanics, pages 1286-1303, 1960.

[10] P. B. Bochev and C. R. Dohrmann. A computational study of stabilized, low-order C0 finite element approximations of Darcy equations. Computational Mechanics, 38(4-5):323-333, 2006.

[11] P. B. Bochev, C. R. Dohrmann, and M. D. Gunzburger. Stabilization of low-order mixed finite elements for the Stokes equations. SIAM Journal on Numerical Analysis, 44(1):82-101, 2006.

[12] R. I. Borja. On the mechanical energy and effective stress in saturated and unsaturated porous continua. International Journal of Solids and Structures, 43(6):1764-1786, 2006.

[13] R. I. Borja. Computational Poromechanics. Lecture Notes, Stanford University, 2014.

[14] R. I. Borja and J. E. Andrade. Critical state plasticity. Part VI: Mesoscale finite element simulation of strain localization in discrete granular materials. Computer Methods in Applied Mechanics and Engineering, 195 (37-40):5115-5140, 2006.

[15] R. I. Borja and A. Koliji. On the effective stress in unsaturated porous continua with double porosity. Journal of the Mechanics and Physics of Solids, 57(8):1182-1193, 2009.

[16] R. I. Borja and C. Tamagnini. Critical state plasticity. Part III: Extension of the infinitesimal model to include finite strains. Computer Methods in Applied Mechanics and Engineering, 155:73-95, 1998.

[17] F. Brezzi. On the existence, uniqueness and approximation of saddlepoint problems arising from Lagrangian multipliers. RAIRO Analyse numérique, 8:129-151.

[18] F. Brezzi and K.-J. Bathe. A discourse on the stability conditions for mixed finite element formulations. Computer Methods in Applied Mechanics and Engineering, 82:27-57, 1990.

[19] F. Brezzi and J. Pitkäranta. On the stabilization of finite element approximations of the stokes equations. In Efficient Solutions of Elliptic Systems, volume 10, pages 11-19. 1984.

[20] C. Burstedde, L. C. Wilcox, and O. Ghattas. p4est: scalable algorithms for parallel adaptive mesh refinement on forests of octrees. SIAM Journal on Scientific Computing, 33(3):1103-1133, 2011. 
[21] C. Callari and F. Federico. FEM validation of a double porosity elastic model for consolidation of structurally complex clayey soils. International Journal for Numerical and Analytical Methods in Geomechanics, 24(4): 367-402, 2000.

[22] A. Carminati, A. Kaestner, O. Ippisch, A. Koliji, P. Lehmann, R. Hassanein, P. Vontobel, E. Lehmann, L. Laloui, L. Vulliet, and H. Flühler. Water flow between soil aggregates. Transport in Porous Media, 68(2): 219-236, 2007.

[23] J. F. Carneiro. Numerical simulations on the influence of matrix diffusion to carbon sequestration in double porosity fissured aquifers. International Journal of Greenhouse Gas Control, 3(4):431-443, 2009.

[24] X. Chateau and L. Dormieux. Micromechanics and saturated and unsaturated porous media. International Journal for Numerical and Analytical Methods in Geomechanics, 26:831-844, 2002.

[25] J. Choo, J. A. White, and R. I. Borja. Hydromechanical modeling of unsaturated flow in double porosity media. International Journal of $\mathrm{Ge}$ omechanics, Accepted.

[26] C. W. Cryer. A comparison of the three-dimensional consolidation theories of biot and Terzaghi. Quarterly Journal of Mechanics and Applied Mathematics, 16:401-412, 1963.

[27] G. Della Vecchia and E. Romero. A fully coupled elasticplastic hydromechanical model for compacted soils accounting for clay activity. International Journal for Numerical and Analytical Methods in Geomechanics, 37:503-535, 2013.

[28] G. Della Vecchia, A-C. Dieudonné, C. Jommi and R. Charlier. Accounting for evolving pore size distribution in water retention models for compacted clays. International Journal for Numerical and Analytical Methods in Geomechanics, in press. DOI:10.1002/nag.2326.

[29] D.P. Do and D. Hoxha. Effective transfer properties of partially saturated geomaterials with interfaces using the immersed interface method. International Journal for Numerical and Analytical Methods in Geomechanics, 37:3237-3257, 2013.

[30] L. Dormieux, A. Molinari, D. Kondo. Micromechanical approach to the behavior of poroelastic materials. Journal of the Mechanics and Physics of Solids, 50:2203-2231, 2002.

[31] C. R. Dohrmann and P. B. Bochev. A stabilized finite element method for the Stokes problem based on polynomial pressure projections. International Journal for Numerical Methods in Fluids, 46:183-201, 2004.

[32] R. C. Dykhuizen. A new coupling term for dual-porosity models. Water Resources Research, 26(2):351-356, 1990.

[33] D. Elsworth and M. Bai. Flow-deformation response of dual-porosity Media. Journal of Geotechnical Engineering, 118(1):107-124, 1992.

[34] H. Fahs, M. Hayek, M. Fahs, and A. Younes. An efficient numerical model for hydrodynamic parameterization in 2D fractured dual-porosity media. Advances in Water Resources, 63:179-193, 2014. 
[35] C.D. Foster, T. Mohammad Nejad. Embedded discontinuity finite element modeling of fluid flow in fractured porous media. Acta Geotechnica, 8:(1):49-57, 2013.

[36] J. Frey, R. Chambon, and C. Dascalu. A two-scale poromechanical model for cohesive rocks. Acta Geotechnica, 8(2):107-124, 2013.

[37] G. N. Gatica, F. J. Sayas. Characterizing the inf-sup condition on product spaces. Numerische Mathematik, 109(2):209-231, 2008.

[38] S. Geiger, M. Dentz, and I. Neuweiler. A novel multi-rate dual-porosity model for improved simulation of fractured and multiporosity reservoirs. SPE Journal, 18(4):670-684, 2013.

[39] H. H. Gerke and M. T. van Genuchten. Evaluation of a first-order water transfer term for variably saturated dual-porosity flow models. Water Resources Research, 29(4):1225-1238, 1993.

[40] A. Giraud, N.B. Nguyen, and D. Grgic. Effective poroelastic coefficients of isotropic oolitic rocks with micro and meso porosities. International Journal of Engineering Science, 58(4):57-77, 2012.

[41] C. Hellmich, D. Celundova and F.-J. Ulm. Multiporoelasticity of hierarchically structured materials: Micromechanical foundations and application to bone. Journal of Engineering Mechanics, 135(4):382-394, 2009.

[42] M. A. Heroux and J. M. Willenbring. A new overview of the Trilinos project. Scientific Programming, 20(2):83-88, 2012.

[43] J. S. Howell and N. J. Walkington. Inf-sup conditions for twofold saddle point problems. Numerische Mathematik, 118:663-693, 2011.

[44] T. J. Hughes, L. Franca, and M. Balestra. A new finite element formulation for computational fluid dynamics: V. Circumventing the BabuškaBrezzi condition: a stable Petrov-Galerkin formulation of the Stokes problem accommodating equal-order interpolations. Computer Methods in Applied Mechanics and Engineering, 59(1):85-99, 1986.

[45] T. J. Hughes, G. Feijóo, L. Mazzei, and J. Quincy. The variational multiscale method - a paradigm for computational mechanics. Computer Methods in Applied Mechanics and Engineering, 166(1-2):3-24, 1998.

[46] L.B. Hu, H. Péron, T. Hueckel and L. Laloui. Desiccation shrinkage of non-clayey soils: multiphysics mechanisms and a microstructural model. International Journal for Numerical and Analytical Methods in Geomechanics, 37:1761-1781, 2013.

[47] N. J. Jarvis. A review of non-equilibrium water flow and solute transport in soil macropores: principles, controlling factors and consequences for water quality. European Journal of Soil Science, 58(3):523-546, 2007.

[48] D. N. Jenkins and J. K. Prentice. Theory for aquifer test analysis in fractured rocks under linear (nonradial) flow conditions. Groundwater, 20(1):12-21, 1982.

[49] D. Katsuki, M. Gutierrez, and A. Almrabat. Stress-dependent elastic wave velocity of microfractured sandstone. International Journal for $\mathrm{Nu}$ merical and Analytical Methods in Geomechanics, 38:441-456, 2014. 
[50] E. Kazatchenko, M. Markov, A. Mousatov, and E. Pervago. Joint inversion of conventional well logs for evaluation of double-porosity carbonate formations. Journal of Petroleum Science and Engineering, 56(4):252266, 2007.

[51] M. Khaled, D. Beskos, and E. Aifantis. On the theory of consolidation with double porosity - III A Finite Element Formulation. International Journal for Numerical and Analytical Methods in Geomechanics, 8:101123, 1984.

[52] J. Kim. Sequential methods for coupled geomechanics and multiphase flow. PhD thesis, Stanford University, 2010.

[53] A. Koliji, L. Laloui, and L. Vulliet. Constitutive modeling of unsaturated aggregated soils. International Journal for Numerical and Analytical Methods in Geomechanics, 34(17):1846-1876, 2010.

[54] A.R. Lamb, G.J. Gorman and D. Elsworth. A fracture mapping and extended finite element scheme for coupled deformation and fluid flow in fractured porous media. International Journal for Numerical and Analytical Methods in Geomechanics, 37(17):2916-2936, 2013.

[55] J. Lewandowska and J. Auriault. Extension of Biot theory to the problem of saturated microporous elastic media with isolated cracks or/and vugs. International Journal for Numerical and Analytical Methods in Geomechanics, 37:2611-2628, 2013.

[56] J. Lewandowska, A. Szymkiewicz, K. Burzyski, and M. Vauclin. Modeling of unsaturated water flow in double-porosity soils by the homogenization approach. Advances in Water Resources, 27(3):283-296, 2004.

[57] J. Lewandowska, A. Szymkiewicz, W. Gorczewska, and M. Vauclin. Infiltration in a double-porosity medium: Experiments and comparison with a theoretical model. Water Resources Research, 41(2):W02022, 2005.

[58] J. Lewandowska, T. T. Ngoc, M. Vauclin, and H. Bertin. Water drainage in double-porosity soils: experiments and micro-macro modeling. Journal of Geotechnical Engineering, 134(2):231-243, 2008.

[59] R. W. Lewis and H. R. Ghafouri. A novel finite element double porosity model for multiphase flow through deformable fractured porous media. International Journal for Numerical and Analytical Methods in Geomechanics, 21(11):789-816, 1997.

[60] L. Li, Q. Meng, S. Wang, G. Li, and C. Tang. A numerical investigation of the hydraulic fracturing behaviour of conglomerate in glutenite formation. Acta Geotechnica, 8(6):597-618, 2013.

[61] F. Liu and R. I. Borja. Stabilized low-order finite elements for frictional contact with the extended finite element method. Computer Methods in Applied Mechanics and Engineering, 199(37-40):2456-2471, 2010.

[62] D. Mašín. Double structure hydromechanical coupling formalism and a model for unsaturated expansive clays. Engineering Geology, 165:73-88, 2013.

[63] A. Moench. Double-porosity models for a fissured groundwater reservoir with fracture skin. Water Resources Research, 20(7):831-846, 1984. 
[64] T. Mohammadnejad and A. R. Khoei. Hydro-mechanical modeling of cohesive crack propagation in multiphase porous media using the extended finite element method. International Journal for Numerical and Analytical Methods in Geomechanics, 37:1247-1279, 2013.

[65] C. Morin and C. Hellmich. A multiscale poromicromechanical approach to wave propagation and attenuation in bone. Ultrasonics, 54:1251-1269, 2014.

[66] M. A. Murad and A. F. D. Loula. Improved accuracy in finite element analysis of Biot's consolidation problem. Computer Methods in Applied Mechanics and Engineering, 95(3):359-382, 1992.

[67] M. A. Murad and A. F. D. Loula On stability and convergence of finite element approximations of Biot's consolidation problem. International Journal for Numerical Methods in Engineering, 37(4):645-667, 1994.

[68] G. Musso, E. Romero, and G. D. Vecchia. Double-structure effects on the chemo-hydro-mechanical behaviour of a compacted active clay. Géotechnique, 63(3):206-220, 2013.

[69] S. K. Ngien, N. A. Rahman, R. W. Lewis, and K. Ahmad. Numerical modelling of multiphase immiscible flow in double-porosity featured groundwater systems. International Journal for Numerical and Analytical Methods in Geomechanics, 36:1330-1349, 2012.

[70] A. Nur and J. D. Byerlee. An exact effective stress law for elastic deformation or rock with fluids. Journal of Geophysical Research, 76(26): 6414-6419, 1971.

[71] M. Preisig and J. H. Prévost. Stabilization procedures in coupled poromechanics problems: A critical assessment. International Journal for $\mathrm{Nu}$ merical and Analytical Methods in Geomechanics, 35(3):1207-1225, 2011.

[72] A. R. Russell. Water retention characteristics of soils with double porosity. European Journal of Soil Science, 61(3):412-424, 2010.

[73] S. Salimzadeh and N. Khalili. Consolidation analysis of unsaturated double porosity media. In: N. Khalili, A. Russell \& A. Khoshghalb (Eds), Unsaturated Soils: Research \& Applications, Taylor \& Francis Group, London, 2014, pp. 575-580.

[74] W. Shen, Z. He, L. Dormieux, and D. Kondo. Effective strength of saturated double porous media with a DruckerPrager solid phase. International Journal for Numerical and Analytical Methods in Geomechanics, 38(3):281-296, 2014.

[75] J. Šimůnek, N. Jarvis, M. T. van Genuchten, and A. Gardenas. Review and comparison of models for describing non-equilibrium and preferential flow and transport in the vadose zone. Journal of Hydrology, 272:14-35, 2003.

[76] X. Song and R. I. Borja. Mathematical framework for unsaturated flow in the finite deformation range. International Journal for Numerical Methods in Engineering, 97:658-792, 2014. 
[77] X. Song and R. I. Borja. Finite deformation and fluid flow in unsaturated soils with random heterogeneity. Vadose Zone Journal, 13(5), 2014. DOI:10.2136/vzj2013.07.0131.

[78] W. Sun, J. T. Ostien, and A. Salinger. A stabilized assumed deformation gradient finite element formulation for strongly coupled poromechanical simulations at finite strain. International Journal for Numerical and Analytical Methods in Geomechanics, 37(16):2755-2788, 2013.

[79] W. Sun, Q. Chen, J. T. Ostien. Modeling the hydro-mechanical responses of strip and circular punch loadings on water-saturated collapsible geomaterials. Acta Geotechnica, 9(5):903-934, 2014.

[80] M. Talebian, R. Al-Khoury, and L. Sluys. A computational model for coupled multiphysics processes of $\mathrm{CO}_{2}$ sequestration in fractured porous media. Advances in Water Resources, 59:238-255, 2013.

[81] C. Taylor and P. Hood. A numerical solution of the Navier-Stokes equations using the finite element technique. Computers \& Fluids, 1:73-100, 1973.

[82] K. Terzaghi. Theoretical Soil Mechanics. Wiley, 1943.

[83] N. Trottier, F. Delay, O. Bildstein, and P. Ackerer. Inversion of a dualcontinuum approach to flow in a karstified limestone: Insight into aquifer heterogeneity revealed by well-test interferences. Journal of Hydrology, 508:157-169, 2014.

[84] A. Truty and T. Zimmermann. Stabilized mixed finite element formulations for materially nonlinear partially saturated two-phase media. Computer Methods in Applied Mechanics and Engineering, 195(13-16):15171546, 2006.

[85] C. Vitone, F. Cotecchia, G. Viggiani, and S. A. Hall. Strain fields and mechanical response of a highly to medium fissured bentonite clay. International Journal for Numerical and Analytical Methods in Geomechanics, 37:1510-1534, 2013.

[86] C. Vitone, G. Viggiani, F. Cotecchia, and S. A. Hall. Localized deformation in intensely fissured clays studied by $2 \mathrm{D}$ digital image correlation. Acta Geotechnica, 8(3):247-263, 2013.

[87] M.-N. Vu, A. Pouya, and D. M. Seyedi. Theoretical and numerical study of the steady-state flow through finite fractured porous media. International Journal for Numerical and Analytical Methods in Geomechanics, 38(3):221-235, 2014.

[88] J. Wan. Stabilized finite element methods for coupled geomechanics and multiphase flow. PhD thesis, Stanford University, 2002.

[89] J. Warren and P. Root. The behavior of naturally fractured reservoirs. SPE Journal, 3(3):245-255, 1963.

[90] J. A. White and R. I. Borja. Stabilized low-order finite elements for coupled solid-deformation/fluid-diffusion and their application to fault zone transients. Computer Methods in Applied Mechanics and Engineering, 197(49-50):4353-4366, 2008. 
[91] J. A. White and R. I. Borja. Block-preconditioned Newton-Krylov solvers for fully coupled flow and geomechanics. Computational Geosciences, 15 (4):647-659, 2011.

[92] R. Wilson and E. Aifantis. On the theory of consolidation with double porosity. International Journal of Engineering Science, 20(9):1009-1035, 1982.

[93] X. Yang, M. C. Richmond, T. D. Scheibe, W. A. Perkins, and H. Resat. Flow Partitioning in Fully Saturated Soil Aggregates. Transport in Porous Media, 103(2):295-314, 2014.

[94] S. Yin. Numerical analysis of thermal fracturing in subsurface cold water injection by finite element methods. International Journal for Numerical and Analytical Methods in Geomechanics, 37(15):2523-2538, 2013.

[95] A. Zaoui. Continuum micromechanics: Survey. Journal of Engineering Mechanics, 128(3):808-816, 2002.

[96] J. Zhang and J.-C. Roegiers. Double porosity finite element method for borehole modeling. Rock Mechanics and Rock Engineering, 38(3):217242,2005 . 